\title{
Nail Psoriasis: A Review of Treatment Options
}

\author{
Marcel C. Pasch $^{1}$ (1)
}

Published online: 4 April 2016

(c) The Author(s) 2016. This article is published with open access at Springerlink.com

\begin{abstract}
Nail involvement affects $80-90 \%$ of patients with plaque psoriasis, and is even more prevalent in patients with psoriatic arthritis. This review is the result of a systemic approach to the literature and covers topical, intralesional, conventional systemic, and biologic systemic treatments, as well as non-pharmacological treatment options for nail psoriasis. The available evidence suggests that all anti-tumor necrosis factor- $\alpha$, anti-interleukin (IL)17 , and anti-IL-12/23 antibodies which are available for plaque psoriasis and psoriatic arthritis are highly effective treatments for nail psoriasis. Conventional systemic treatments, including methotrexate, cyclosporine, acitretin, and apremilast, as well as intralesional corticosteroids, can also be effective treatments for nail psoriasis. Topical treatments, including corticosteroids, calcipotriol, tacrolimus, and tazarotene, have also been shown to have a position in the treatment of nail psoriasis, particularly in mild cases. Finally, non-pharmacological treatment options, including phototherapy, photodynamic therapy, laser therapy, and several radiotherapeutic options, are also reviewed but cannot be advised as first-line treatment options. Another conclusion of this review is that the lack of a reliable core set of outcomes measures for trials in nail psoriasis hinders the interpretation of results, and is urgently needed.
\end{abstract}

Marcel C. Pasch

marcel.pasch@radboudumc.nl

1 Department of Dermatology, Radboud University Medical Center, PO Box 9101, 6500 HB Nijmegen (370), The Netherlands

\section{Key Points}

Nail psoriasis can be treated effectively using topical treatments, intralesional treatments, and systemic treatments, but an optimal effect may take up to 1 year.

The role of non-pharmacological treatment options, including phototherapy, photodynamic therapy, and laser therapy, is limited.

An undesirable heterogeneity of outcome measures and scoring systems makes it almost impossible to compare trials.

\section{Introduction}

Psoriasis is a common inflammatory skin disease that causes significant stress and morbidity. It most often presents with well-demarcated, scaling and erythematous plaques, often at the extensor surfaces of knees and elbows. The prevalence varies between 0.7 and $2.9 \%$, with a preference for the Caucasian population. Plaque psoriasis (PP, or psoriasis vulgaris) is the most common form of the disease, affecting $85-90 \%$ of patients, and manifests with patches on the trunk and extremities. Other common forms of psoriasis may affect the scalp, joints, creases, or nails, even in patients without psoriasis of the skin.

Among PP patients, prevalence of nail psoriasis documented in the literature is over $50 \%$, with an estimated lifetime incidence of $80-90 \%$ [1]. A recent survey by Klaassen et al. found nail involvement in $66.0 \%$ of 1459 
psoriasis patients, which indicates that the prevalence of nail psoriasis might often be underestimated [2]. Among patients with psoriatic arthritis (PsA), the prevalence of nail psoriasis may be $>80 \%$ [3]. Nail psoriasis in the absence of cutaneous or joint disease is present in 5-10\% of psoriatic patients [4]. Psoriatic nail disease may be considered an indicator for patients at risk for future psoriatic joint damage $[5,6]$.

Nail psoriasis may show different clinical presentations according to the structure that is involved within the nail apparatus. All signs of nail psoriasis are not specific and may be found in several other nail conditions. Therefore, histology of involved tissue is the gold standard for making the diagnosis of nail psoriasis; however, in most cases, the diagnosis of nail psoriasis can be made clinically by pattern recognition. When psoriasis is present in the nail-forming unit (the nail matrix), it can cause the following manifestations: pitting, leukonychia (white spots within the nail plate), red spots of the lunula, transverse grooves (Beau's lines), and crumbling of the nail plate (Fig. 1). Psoriasis of the nail bed presents as oil-drop discoloration, splinter hemorrhages involving the distal third of the nail plate, subungual hyperkeratosis, and/or detachment of the nail plate from the nail bed (onycholysis). Psoriasis can also involve the periungual region, resulting in psoriatic paronychia. Looking at psoriatic nails, it is important to evaluate the contribution of nail matrix disease and nail bed disease separately because some treatment options have a better effect on matrix disease, while others are more efficient in treating nail bed disease.

It is known that psoriasis on visible areas of the skin, such as the face and hands, may have a substantial negative impact on physical, psychological, and social dimensions of quality of life (QoL) [7-11]. In addition, fingernail psoriasis is highly visible and has a relevant and additional negative impact on the QoL of psoriasis patients, particularly in patients with both nail matrix and nail bed signs of the disease [12-14]. Patients with only nail bed alterations scored significant lower QoL scores when compared with patients with only nail matrix features. The additional negative consequences of nail involvement in psoriasis on QoL may be explained by the fact that nail psoriasis is more than a highly visible variant. Complaints of patients with nail psoriasis include pain, inability to grasp small objects, tie shoe laces or button clothes, and cause an altered sense of fine touch. Pain in nail psoriasis has a high association with joint pain, therefore the presence of nail psoriasis may identify patients who are at risk of developing disabling PsA [13].

The impact of nail psoriasis on individual patients can be high. A recent survey showed that $47 \%$ of patients with nail psoriasis would like to receive treatment for their nail disorder [2]; however, treatment of nail psoriasis is challenging, and involves topical, intralesional, and systemic

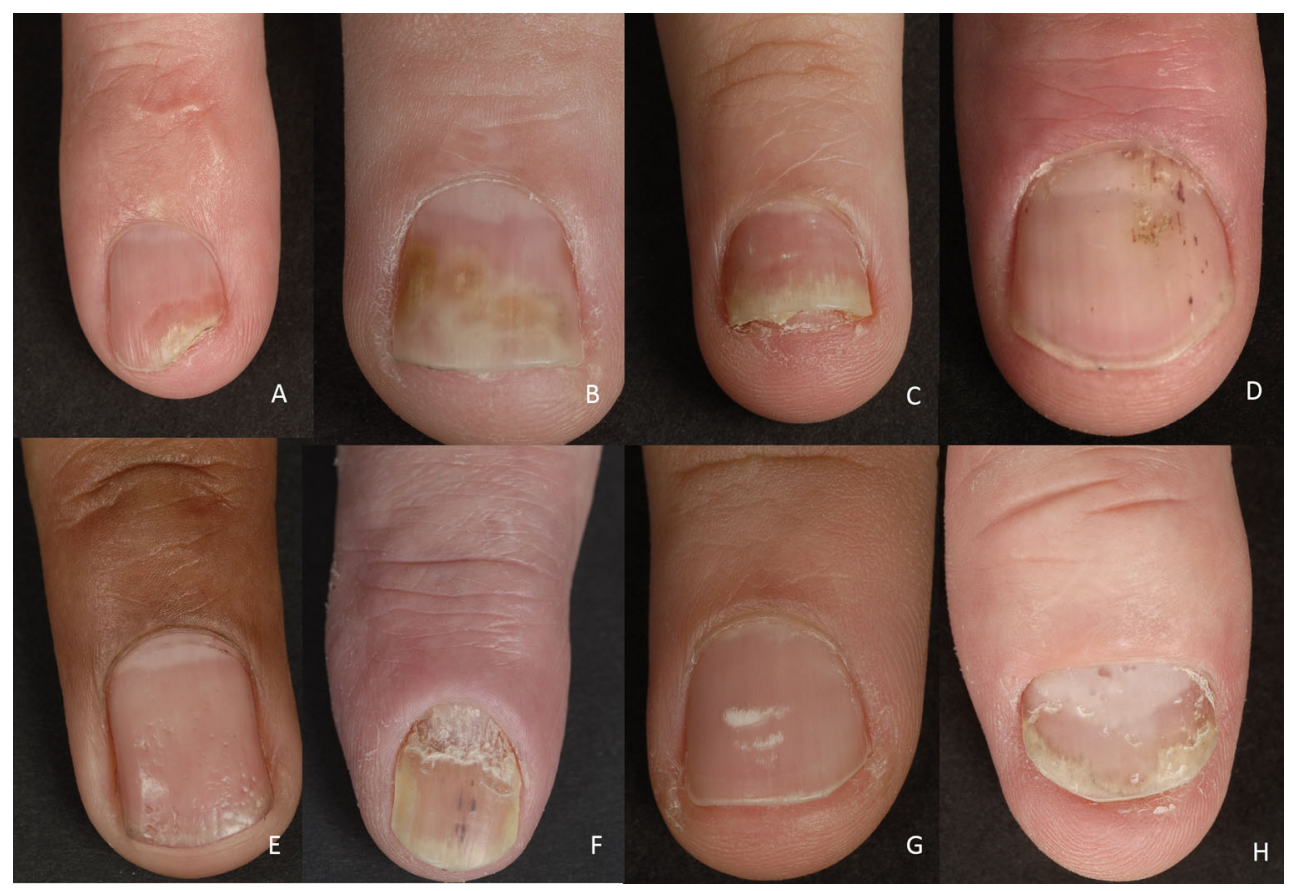

Fig. 1 Nail manifestations seen in nail psoriasis. Nail bed features a oil-drop discoloration, b onycholysis, $\mathbf{c}$ subungual hyperkeratosis, d splinter hemorrhages. Nail matrix features e pitting of the nail plate, f crumbling in proximal quadrants of the nail plate, $\mathbf{g}$ leukonychia, $\mathbf{h}$ red spot in the lunula. Courtesy of K. Klaassen 
therapies. A recent Cochrane review discussed randomized controlled trials (RCTs) on nail psoriasis [15]. The practical use of that review is limited by the fact that most studies on nail psoriasis are largely anecdotal, case-series, or derived from open-label, prospective studies. Therefore, several frequently used, and considered effective, treatment options are not discussed in that Cochrane review. The aim of that review was to cover all published data on the treatment of nail psoriasis, including not only data from RCTs but also from other studies and case reports.

\section{Pathophysiology}

Psoriatic lesions highlight the fundamental processes underlying its pathogenesis, namely inflammation and epidermal hyperproliferation. Psoriasis appears to be a multifactorial disease whose exact underlying mechanism is still unclear, but environmental factors, genetic susceptibility, abnormal function of keratinocytes, and immunological disturbances of the innate and acquired immune system are all postulated [16, 17].

Environmental factors that have been identified as triggering or exacerbating psoriasis in susceptible persons are certain infections, drugs, stressful life events, and smoking. The role of genetic factors has been a matter of research, particularly in the past decades. Population studies have shown that the incidence of psoriasis is greater among firstand second-degree relatives of psoriasis patients than among the general population [18]. Genome-wide association studies have identified nine chromosomal loci (PSORS1 through PSORS9) that can be linked to psoriasis. The major genetic determinant seems to be PSORS1, which probably accounts for $35-50 \%$ of the hereditary component of psoriasis. Human leukocyte antigen (HLA)Cw6, involved in antigen presentation, seems to be the susceptible allele located on PSORS1. However, patients with nail psoriasis and/or PsA are more frequently HLACw6-negative, indicating a separate genotype [19]. In recent years, several other genome-wide association studies identified genes related to the skin barrier function and to both the innate and adaptive immune system [20-22].

Over the years, various cells and mediators playing a role in the immunopathogenesis of psoriasis have been identified or postulated, including keratinocytes, dendritic cells, T lymphocytes, complement proteins, and many cytokines and chemokines. Briefly, in the current concept an initial trigger (e.g. trauma, infection, and stress) can induce the production of various cytokines (including tumor necrosis factor[TNF]- $\alpha$ ) by innate immune cells, resulting in a cascade of events eventually leading to the activation of dendritic cells [16]. Activated dendritic cells start to secrete mediators (e.g. interleukin [IL]-12, IL-23) and present antigens to $\mathrm{T}$ cells, leading to the differentiation of type 1 and type 17 helper T cells (Th1, Th17). These activated $\mathrm{T}$ cells subsequently secrete mediators (IL17A, IL-17F, and IL-22), which then activate keratinocytes, resulting in keratinocyte hyperproliferation and the secretion of different chemokines by these keratinocytes. Keratinocyte hyperproliferation is responsible for the clinical feature of scaling, while the released chemokines are responsible for attraction of more inflammatory cells to the skin. This model of pathogenesis of psoriasis has resulted in the production of monoclonal antibodies interfering in several pathogenic steps, the socalled biologics, which prevent the expression of the full pathogenic cascade, and thus reducing the clinical features of psoriasis.

\section{Assessment of Nail Psoriasis}

The availability of sensitive, responsive, specific, and validated outcome measures is essential in drug research and comparison of trials. Unfortunately, these are still missing in nail psoriasis. Most recent studies on nail psoriasis use the Nail Psoriasis Severity Index (NAPSI), target NAPSI, or one of its many variants as a marker of nail improvement [23]. To calculate the NAPSI, each nail is divided into four quadrants, each of which are evaluated for the presence of any manifestations of psoriasis in the nail matrix (pitting, leukonychia, red spots in the lunula, nail plate crumbling) and nail bed (oil-drop discoloration, onycholysis, hyperkeratosis, splinter hemorrhages). If any sign is present in all four quadrants, the nail is given a score of 4 , through to a score of 0 if there are no signs in any quadrant. Each nail is assigned a nail matrix and a nail bed score of $0-4$, which are combined to yield a total score of $0-8$ for each nail. All nails may be evaluated, with the total NAPSI score being the sum of the scores, up to 80 if only fingers (10 nails) are considered, or up to 160 if toes are also included (20 nails). In clinical studies, often only the most seriously involved nail is targeted (target NAPSI) to assess the effects of drug therapy. Some studies use (target) NAPSI-50, NAPSI-75, and NAPSI-90 to indicate the percentage of patients achieving a (target) NAPSI improvement of at least 50,75 , or $90 \%$, respectively. Despite its current popularity in nail psoriasis studies, the NAPSI has some disadvantages. Its poor validation, the fact that the important nail psoriasis feature 'subungual hyperkeratosis' is not included and, in particular, the lack of correspondence of NAPSI scores with the clinical severity of nail psoriasis are important disadvantages of this scoring system [24]. Most of these limitations are absent in a newly developed scoring system, the N-NAIL, which has been partly validated [24]; however, this system has not been 
used in any published clinical studies. Older studies have used many different scoring systems, which, together with the major differences in study design, inclusion criteria, and follow-up, make it difficult to compare the results of individual trials. Furthermore, not only should clinical severity be part of a core outcome set in nail psoriasis but, ideally, it should also involve patient-assessed signs, QoL, patient satisfaction with treatment and outcome, adverse events, and probably costs. A first attempt at developing a tool for the assessment of various domains of nail psoriasis outcomes, known as Nail Assessment in Psoriasis and Psoriatic Arthritis (NAPPA), has recently been published [25].

\section{Management of Nail Psoriasis}

Treating nail psoriasis is often a time-consuming challenge with an unsecure outcome. Response to treatment may appear everywhere in the spectrum, from very disappointing to excellent, but relapses are common. Unfortunately, there is a lack of evidence-based treatments and consequent guidelines [15]. This does not necessarily mean that available treatments are not efficacious, but that final statistical evidence is missing. Therapeutic options include patient education, external topical treatments, intralesional steroids, systemic treatments, and non-pharmacological treatment options. Patient education should cover the avoidance of minor repetitive nail trauma, managing expectations with regard to the amount of time necessary for nail clearing, and discussing prevention and treatment of fungal infections in psoriasis nails [26]. Psoriatic nail changes can be provoked by minor traumas, such as nail biting, subungual cleaning, picking, or manicure. Patients should avoid these habits and keep the nails as short as possible to prevent trauma or lifting of the poorly attached nail plate [27]. Before the start of treatment, the clinician should discuss with the patient that any noticeable nail improvement will take a long time; many treatments will show maximal results only after 1 year. The low growth rate of the nail plate is responsible for a delay of 3-9 months before clinical improvement can be noticed in cases of effective treatment. Four to 6 months is a reasonable period of treatment before evaluating clinically relevant results. In the beginning, the improvement may be so limited that it is advisable to take photographs of the nails during each visit to convince both the patient and the physician that the treatment has positive results. Concomitant onychomycosis has a high prevalence in psoriatic nails. A meta-analysis has shown a prevalence of $18.0 \%$ in psoriasis patients compared with a prevalence of $9.1 \%$ in control groups [28]. This high prevalence rate should be a reason for excluding onychomycosis before starting intensive treatment for nail psoriasis. Obviously, treatment of concomitant onychomycosis will improve the outcome of all other treatments.

The choice of treatments depends on clinical presentation, as well as patient-related factors. Most patients have only mild nail psoriasis without signs of PsA or severe PP. These patients may profit from topical treatment, while systemic treatment is indicated in patients with severe nail psoriasis, major impact on QoL, or concomitant moderate to severe psoriatic skin lesions. Systemic therapy should also be favored if concomitant PsA is evident. The choice of treatments further depends on patient factors, including age, experienced burden of disease, accompanying diseases and therapies, individual patient preferences, and the risks of treatment.

This review covers treatment options for nail psoriasis and is the result of a systemic approach to the literature. The following databases were searched (to December 2015): the Cochrane Skin Group Specialised Register, CENTRAL in The Cochrane Library, and Pubmed (from 1948). Essentially, the search strategy included psoria*, and (nail*, or toenail* or thumbnail*, or ungual, or unguel, or unguium, or pitting, or punctate, or Beau's lines, or subungual hyperkeratosis, or onycholysis) and (trial). Overall, 120 publications were identified using this search strategy. The titles and abstracts of each of these publications were evaluated and a total of 72 studies or case reports on the treatment of nail psoriasis were selected for complete reviewing. Publications without original data were discarded. In addition, the reference lists of all publications were checked, which resulted in an 69 additional publications. The number of additional publications retrieved by checking reference lists was high, and was mainly caused by the fact that nail psoriasis was often a secondary endpoint in studies on PP or PsA, and was not mentioned in the abstract.

\section{Topical Treatment Modalities}

Penetration of a pharmacological agent into the site of psoriatic inflammation, the nail bed, or the nail matrix is essential to achieve therapeutic concentrations. Given the anatomical structure and physical characteristics of the nail, it is difficult, or impossible, for antipsoriatic agents to penetrate through the nail plate to the site of psoriatic inflammation. To overcome this contrast of desired penetration and an impermeable physical barrier caused by the nail plate, it is essential to distinguish signs of nail matrix psoriasis from signs of nail bed psoriasis (Fig. 1). When signs of nail matrix psoriasis are present, the topical medication should be applied on the proximal nail fold, i.e. just above the nail matrix. If signs of nail bed psoriasis are 
present, the compound should be able to penetrate to the psoriatic inflammation of the nail bed. Therefore, the onycholytic nail should be trimmed as much as possible before and during treatment to allow the application of the compound to the nail bed.

\subsection{Topical Corticosteroids}

Topical corticosteroids have been used for many decades to suppress psoriatic inflammation in PP. Also in nail psoriasis, potent and superpotent corticosteroids are used frequently, and appear to be more effective in nail matrix psoriasis than in nail bed psoriasis. Topical corticosteroids are available in the form of ointments, creams, lotions, emulsions, and even nail lacquers, and the frequency of application frequency is usually once or twice daily. If used frequently and for a prolonged period, telangiectasia of the surrounding skin and atrophy of the skin and underlying phalanx may appear. Others prefer pulse therapy, with application on four consecutive days each week, in order to prevent these side effects. Clinical improvement may not be expected within 4-6 months of treatment, and further improvement can be seen during the first year.

In spite of the long history of corticosteroids in the treatment of nail psoriasis, only a few formal trials of their efficacy have been conducted. Clinical studies on topical corticosteroid monotherapy are even sparser. RCTs using betamethasone dipropionate ointment $(64 \mathrm{mg} / \mathrm{g})$ twice daily $[29,30]$ and clobetasol propionate $0.05 \%$ cream under occlusion once daily [31] identified positive effects on subungual hyperkeratosis, salmon patches, pitting, and onycholysis after 12-20 weeks of use. One study compared betamethasone dipropionate $(64 \mathrm{mg} / \mathrm{g})$ and salicylic acid $(0.03 \mathrm{~g} / \mathrm{g})$ ointment with calcipotriol ointment $(50 \mu \mathrm{g} / \mathrm{g})$ in the treatment of nail bed psoriasis with severe subungual hyperkeratosis [30]. After 3 months of treatment, subungual fingernail and toenail hyperkeratosis was reduced by $20-30 \%$ in both groups (no statistical differences). Rigopoulos et al. conducted an RCT comparing tazarotene $0.1 \%$ cream with clobetasol propionate $0.05 \%$ cream under occlusion once daily for 12 weeks [31]. Patients showed a significant improvement for pitting, onycholysis, hyperkeratosis, and salmon patches with both agents, without significant differences between the two investigated agents. Mild side effects were reported in $18.8 \%$ of the tazarotene-treated patients, including desquamation and erythema of the nail-fold skin, periungual irritation, paronychia, and irritation of the skin of the toe or finger distanced from the nail area. Formulations with corticosteroids in a nail lacquer have been studied more often than other forms but are not yet commercially available. In particular, $8 \%$ clobetasol-17-propionate in a nail lacquer has shown positive results in placebo-controlled, open studies [32-34]. Fifty-two to $90 \%$ of patients showed improvement after therapy, which was directly related to the length of treatment. The symptoms that responded best to therapy were onycholysis and pitting (indicating an action on the nail matrix); however, improvement of salmon patches and subungual hyperkeratosis has also been reported, whereas splinter hemorrhages were most persistent. No adverse effects, such as atrophy, hypochromy, periungual telangiectasia, local pain, and hypersensitivity, were noted.

\subsection{Combination Therapy with Corticosteroids}

The assumption that topical corticosteroids in nail psoriasis are particularly effective in ameliorating nail matrix signs of this disease has resulted in several studies in which topical steroids were combined with treatments that are presumed to be more effective in nail bed signs of nail psoriasis. In particular, the combination of topical steroids (clobetasol propionate and betamethasone dipropionate) with the vitamin D3 analog calcipotriol $(50 \mu \mathrm{g} / \mathrm{g})$ has been investigated and appears to be effective [35-37]. This combination is currently one of the most widely used topical treatmentsfor psoriatic nail disease. In one study, calcipotriol cream was used every night five times per week, clobetasol propionate cream was used two times per week for 6 months, and patients were followed-up for a further 6 months, using only clobetasol propionate cream two nights per week [35]. After 6 months of treatment, the mean reduction in hyperkeratosis of the fingernails and toenails was 72.3 and $69.9 \%$, respectively, and at month 12 the decrease was 81.2 and $72.5 \%$, respectively. Another study compared once-daily calcipotriol $(50 \mu \mathrm{g} / \mathrm{g})$ combination therapy with betamethasone dipropionate $(0.05 \%)$ with twice-daily calcipotriol $(50 \mu \mathrm{g} / \mathrm{g})$ monotherapy in the treatment of nail psoriasis for 12 weeks [36]. A similar reduction in target NAPSI was noted in both groups, mainly caused by the improvement of oil-drop discoloration, because other nail bed and matrix features failed to improve with either treatment. A third study used calcipotriol plus betamethasone dipropionate twocompound ointment once daily [37]. At the end of the 12 weeks of treatment, NAPSI showed a $72 \%$ improvement; however, mean NAPSI at baseline was 5.8 , indicating an extremely mild nail psoriasis at baseline [38]. Adverse events included mild irritation on the fingertips of two patients, which was self-limited.

Tacalcitol is another chemical vitamin D analog that has been investigated in a small, open study with both nail bed and nail matrix psoriasis. Patients were treated with a nail lacquer containing $8 \%$ clobetasol-17-propionate applied at bedtime during the weekend, and with tacalcitol $0.1 \%$ ointment under occlusion on the remaining days, for 
6 months [39]. Clinical characteristics of nail bed and nail matrix psoriasis improved markedly, and target NAPSI had improved $50 \%$ at 3 months, and $78 \%$ at 6 months.

\subsection{Intralesional Corticosteroids}

Locally injected steroids have a long history in the treatment of nail psoriasis [40]. Locally penetrating doses of steroid into a digit are administered by needle injection or highpressure jet from a tool such as the 'Port-o-jet', 'Med-Jet MBX', or others. Needle injection is the most common of these, with the temporary popularity of the Port-o-jet waning as concern developed over the potential for splash-back of blood and steroid from the breached epidermis on to the instrument and practitioner, or the development of epidermal inclusion cysts. The existing evidence suggests that intralesional injection into the nail bed and matrix are particularly effective for alleviating lesions caused by psoriasis of the nail matrix, and also has moderate effects on nail bed signs (Table 1). Injections in the region of the nail matrix has almost no effects on nail bed signs of nail psoriasis.

The recommended protocols of treatment differ considerably. Initially, injections of triamcinolone acetonide $(5 \mathrm{mg} / \mathrm{ml})$ were administered monthly for 6 months in the proximal nail fold, followed by a further four injections over the next 6 months and then every 2 months for the final 6- to 12-month period [40-43]. More recent publications prefer a higher concentration of triamcinolone acetonide $(10 \mathrm{mg} / \mathrm{ml}), 0.1 \mathrm{ml}$ administered in each of the four periungual sites, ensuring symmetrical delivery of the steroid to the nail matrix and nail bed, and administered less frequently, such as every 2 months [44, 45]. Clinical results of this modified regimen were investigated in two studies suggesting improved efficacy on the signs of nail bed psoriasis (Table 1) [44, 45].

Side effects after these procedures are well known: short-term paresthesia $[44,45]$ and focal pain that may last for several months [40, 45]; hematoma formation is rather common (up to $20 \%$ ) but asymptomatic [45]. Loss of the nail plate was seen in $9 \%$ of patients in one study [45]. Occasionally, nail-fold atrophy can be encountered, and is often reversible. Chronic topical therapy can lead to complications of the 'disappearing digit' [46] with atrophy of the underlying phalanx [47]. Rupture of the extensor tendon has also been reported after local injection of steroids $[48,49]$.

\subsection{Vitamin D3 Derivates: Calcipotriol, Tacalcitol, Calcitriol}

Vitamin D analogs regulate epidermal cell proliferation and differentiation, as well as production and release of proinflammatory cytokines. Topical formulations containing vitamin D3 (calcitriol) and vitamin D analogs (calcipotriol, tacalcitol) are effective treatments for PP, both as monotherapies and in fixed combinations with corticosteroids. Vitamin D3 derivates appear to be more effective in treating nail bed signs of psoriasis than in nail matrix signs. Studies using calcitriol in the treatment of nail psoriasis are rare. One case was reported whereby the patient responded well to treatment with calcitriol ointment [50]; however, it was not completely clear whether this patient suffered from nail psoriasis. One RCT compared calcitriol ointment $(3 \mu \mathrm{g} / \mathrm{g})$ twice daily with betamethasone diproprionate $(64 \mathrm{mg} / \mathrm{g})$ on subungual hyperkeratosis [29]. After 20 weeks, nails treated with calcitriol had a $38 \%$ reduction in thickness compared with a $35 \%$ reduction seen in nails treated with betamethasone diproprionate (not significant).

Tacalcitol is a chemical vitamin D analog that has been investigated as a monotherapy in one open study of 15 patients with both nail bed and nail matrix psoriasis [51]. Patients used the tacalcitol ointment $(4 \mu \mathrm{g} / \mathrm{g})$ every night in occlusion (with cotton gloves) for 6 months. After 3 months, the NAPSI dropped more than $50 \%$, and after 6 months of treatment the NAPSI was approximately $75 \%$ lower than at baseline. Most patients presented with pain at the beginning of the study, but none reported discomfort after 6 months. Surprisingly, the greatest improvement was seen in nail matrix signs of nail psoriasis. Combining tacalcitol ointment $(10 \mu \mathrm{g} / \mathrm{g})$ with a nail lacquer containing $8 \%$ clobetasol-17-propionate (twice weekly) resulted in major improvement of both nail bed and nail matrix signs [39].

Calcipotriol is the vitamin D analog most widely investigated in nail psoriasis, often in combination with other treatments but also as monotherapy. One case-series study evaluated the efficacy of calcipotriol ointment $(50 \mu \mathrm{g} / \mathrm{g})$ monotherapy twice daily without occlusion in the treatment of nail psoriasis for 5 months [52]. Calcipotriol was particularly effective in nail bed signs of subungual hyperkeratosis, onycholysis, and discoloration. In addition, fingertip tenderness and pain of an involved distal phalanx were significantly reduced. In a 12-week study comparing calcipotriol $(50 \mu \mathrm{g} / \mathrm{g})$ monotherapy with calcipotriol/betamethasone-dipropionate combination therapy, only oil-drop discoloration improved, without differences between the two groups [36]. The same two compounds in a single ointment resulted in a $72 \%$ NAPSI improvement at 12 weeks in patients with extremely mild nail psoriasis [37]. Other studies of combination therapy of calcipotriol and corticosteroids are discussed in Sect. 5.2.

The combination of calcipotriol with oral cyclosporine was used in a study in which a combination of cyclosporine and topical calcipotriol cream versus cyclosporine alone was evaluated [53]. Patients were treated for 3 months with 


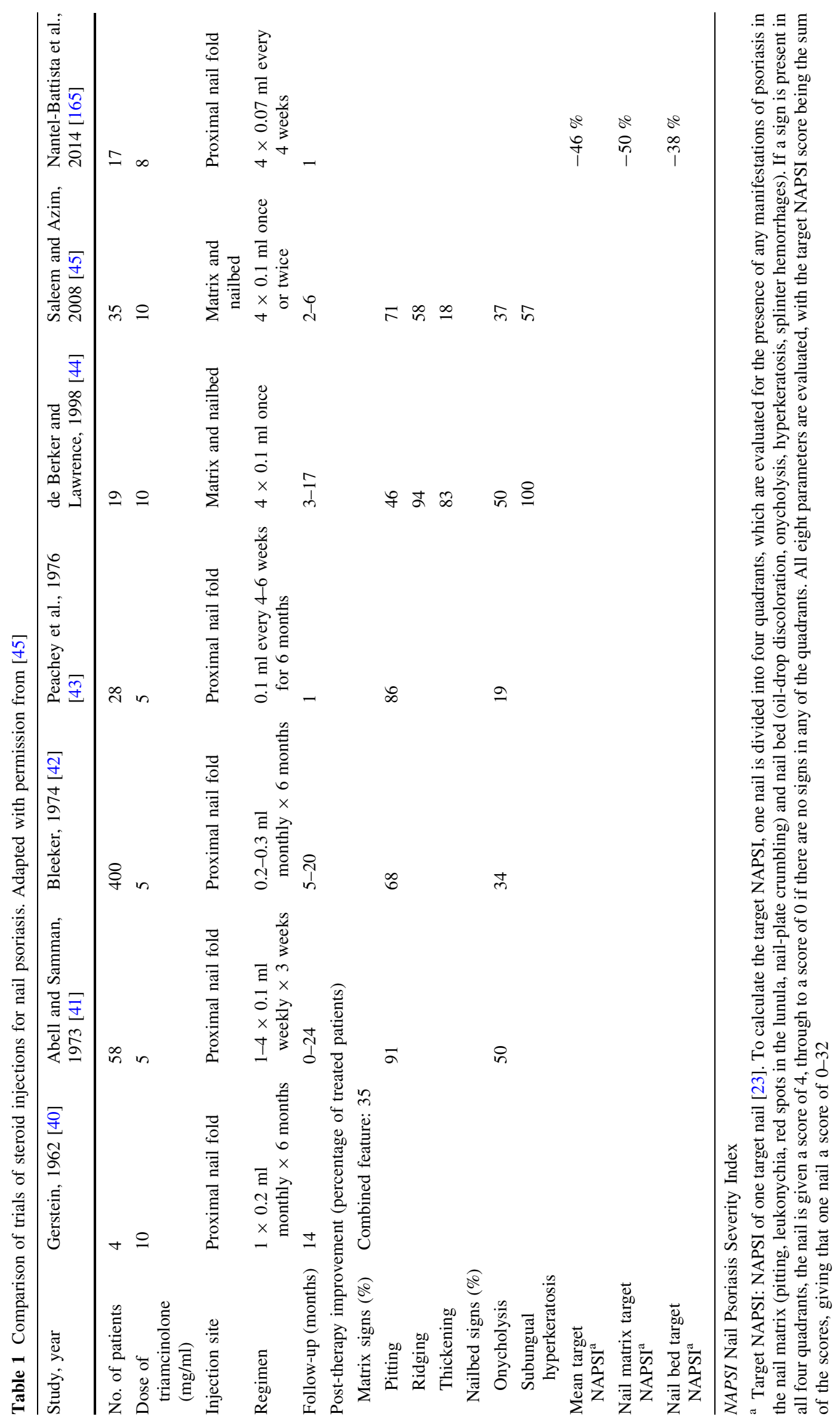


cyclosporine alone (3-4.5 mg/kg/day) or with cyclosporine plus topical application of calcipotriol cream twice daily (with occlusive application at night). Ungual hyperkeratosis, onycholysis, and pitting improved in approximately $80 \%$ of patients using cyclosporine plus calcipotriol, and in approximately $50 \%$ of patients in the cyclosporinealone group, emphasizing the positive effect of vitamin D derivates on nail bed psoriasis.

\subsection{Tazarotene}

Tazarotene is a member of the acetylenic class of retinoids that is selective for $\beta$ and $\gamma$ isotypes of the retinoic acid receptor. It has been shown to have antiproliferative, normalizing-of-differentiation, and anti-inflammatory effects, and is approved for the treatment of psoriasis, acne, and photodamage. Several studies have been performed to estimate efficacy in nail psoriasis [31, 54-57]. In these studies, tazarotene $0.1 \%$ gel, ointment, or cream was used once daily for 12-24 weeks. In one study, tazarotene was effective for onycholysis and pitting (only if applied under occlusion) at 24 weeks, but not for subungual hyperkeratosis, leukonychia, nail plate crumbling/loss, or splinter hemorrhages [54]; however, other studies saw an improvement in hyperkeratosis and oil spots [55, 58]. A better response of nail bed signs than nail matrix signs was also concluded from another study [56]. An $88 \%$ improvement in NAPSI at 6 months was reported from this study, but patients had only mild nail psoriasis at baseline (median NAPSI 15). Rigopoulos et al. conducted a randomized, double-blind study comparing tazarotene cream with clobetasol propionate $0.05 \%$ cream, both under occlusion [31]. The results of this study showed a significant time-effect improvement for pitting, onycholysis, hyperkeratosis, and salmon patches with both agents, without significant differences between the two investigated agents. The most recent study investigating tazarotene in nail psoriasis compared the efficacy of pulsed dye laser (PDL) plus topical tazarotene cream versus topical tazarotene alone [57]. After 6 months of tazarotene treatment without occlusion, no improvement in nail bed or nail matrix psoriasis could be observed. An explanation for this failure might be the fact that most patients were also receiving systemic therapy or using phototherapy. The laser results will be discussed in Sect. 10.1.

From these studies, tazarotene appears to be effective for nail bed signs of psoriasis only. Use may be limited by the preferred use under occlusion and by the relatively frequently occurring side effects, such as erythema, irritation, desquamation, and paronychia.

\subsection{Topical Calcineurin Inhibitors}

Calcineurin is a serine/threonine protein phosphatase that activates T cells by upregulation of the expression of IL-2. Increased IL-2 stimulates the growth and differentiation of $\mathrm{T}$ cell responses. Systemic inhibition of calcineurin, particularly with cyclosporine, has been shown to be an effective treatment for PP [59]. The use of systemic calcineurin inhibitors in psoriasis is limited by potential severe side effects; however, topical treatment of nail psoriasis with calcineurin inhibitors could prevent the majority of these side effects. Several case reports and studies using topical cyclosporine and tacrolimus have been published [60-62]. In a prospective, randomized, placebo-controlled study, nails of eight patients were treated twice daily for 12 weeks with a $70 \mathrm{mg} / \mathrm{ml}$ maize-oil-dissolved oral cyclosporine solution [61]. This study showed excellent results in the majority of patients; mean improvement was $77 \%$ in the cyclosporine-treated group and $12 \%$ in the placebo-treated group. Improvement was noted both in nail matrix signs of nail psoriasis (pitting) and in nail bed signs (hyperkeratosis, onycholysis, crumbling, and oil-drop discoloration). No local or systemic side effects were detectable, but nails may turn yellowish after long-term application of cyclosporine in maize oil. These positive results were rather surprising considering the known issue of difficult penetration of cyclosporine through the skin and the nail due to its highly lipophilic nature and size of the molecule [63]. Later, Prins et al. reported pharmaceutical instability of the used topical cyclosporine emulsion for nail psoriasis within $4 \mathrm{~h}$ after preparation [64]. According to this study, patients should homogenize the emulsion before each application in order to prevent treatment failure. An unpublished study from Rotterdam, The Netherlands, sponsored by Novartis (ISRCTN47031769) tried to confirm the positive results of cyclosporine on nail psoriasis, but the investigators were unable to find differences between the cyclosporine-treated nails and the maize-oiltreated nails (personal communication).

Tacrolimus is a calcineurin inhibitor with much better skin-penetrating capacities than cyclosporine. In a 12-week randomized, controlled, open-label study, the efficacy and safety of topical treatment with tacrolimus $0.1 \%$ ointment was assessed in 21 patients with nail psoriasis [62]. Application was once daily at bedtime onto the nail folds of affected nails, without occlusion, and patients were instructed not to wash their hands until the next morning. The severity of nail psoriasis improved significantly, as measured by overall NAPSI $(-57 \%)$ and target NAPSI $(-65 \%)$. Tacrolimus ointment proved to be equally effective on nail bed and nail matrix features. One patient 
was withdrawn from tacrolimus application after 9 weeks because of the appearance of acute paronychia. No other side effects were recorded in the observation time.These results suggest that topical tacrolimus is one of the more effective and tolerable treatments for both nail bed and nail matrix psoriasis, but these results need confirmation from additional studies.

\subsection{Anthralin}

Anthralin (dithranol) was first synthesized as a derivative of chrysarobin, prepared from the araroba tree in Brazil over a century ago. It is an effective treatment of skin lesions in psoriasis by inhibiting keratinocyte hyperproliferation and interfering with several relevant steps of the immune system [65]. In one uncontrolled study, 20 psoriasis patients with nail involvement were treated with topical anthralin therapy [66]. An ointment of anthralin in petrolatum was applied to the affected nail bed once daily and then washed away with water after $30 \mathrm{~min}$. Therapy was started with $0.4 \%$ anthralin, and in case of lack of improvement, the concentration was gradually increased up to $2.0 \%$. Within 5 months of therapy, approximately half of the patients showed good improvement of nail bed signs of onycholysis, hyperkeratosis, and thickening of the nail. In addition, pitting, a nail matrix sign of nail psoriasis, had improved, which is surprising since only the nail bed had been treated. Longitudinal lines or transverse lines were unresponsive. A disturbing side effect of anthralin therapy was reversible pigmentation of the nail plate, in particular accumulating in the pits. This hyperpigmentation could be reduced, but not abolished, with $10 \%$ triethanolamine cream applied after washing away the anthralin cream.

\subsection{5-Fluorouracil}

5-fluorouracil is a chemotherapeutic agent with antimitotic and cytotoxic effects that is efficacious for a number of dermatological disorders, primarily those that result from keratinocyte hyperproliferation, including superficial basal cell carcinoma and actinic keratosis. 5-Fluorouracil in several vehicles has had variable results in the treatment of nail psoriasis [67-69]. Most studies showed no or very poor response [67, 69], but Fritz et al. reported an improvement of more than $50 \%$ in the clinical signs of nail psoriasis after treatment with $20 \%$ urea plus $1 \% 5$-fluorouracil cream over a period of 6 months [68]. Common treatment-related adverse effects were inflammation, infection, discoloration, and onycholysis. 5-Fluorouracil is not used commonly in nail psoriasis because of its frequent adverse effects and doubtful efficacy.

\subsection{Miscellaneous Topical Therapies: Allopurinol, Intralesional Methotrexate, Colloidal Silicic Acid, Indigo Naturalis Extract}

Allopurinol, intralesional methotrexate, colloidal silicic acid, and indigo naturalis extract have been investigated in nail psoriasis but are not routinely used in clinical practice because of insufficient data, poor study results, or lack of registration. Allopurinol is a purine analog and an oftenprescribed urate-lowering drug for the management of gout. It inhibits xanthine oxidase, a key enzyme involved in the oxidation of hypoxanthine and xanthine, reactions that ultimately result in the production of uric acid. Case reports from the early 1970s suggested a positive effect of allopurinol on PP. One placebo-controlled study investigated allopurinol (200 mg three times daily) in PP and nail psoriasis [70]. Allopurinol was found to be no more effective than placebo after 8 weeks of treatment.

Methotrexate is a folic acid analog that irreversibly binds to dehydrofolate reductase and blocks deoxyribonucleic acid synthesis. It is usually taken orally or administered by injection (intramuscular, intravenous, subcutaneous) and has several indications, including psoriasis. Intralesional use of methotrexate may limit the dose, and hence the side effects, but is not a generally accepted treatment in nail psoriasis. Saricaoglu et al. reported a 26-year-old women who was treated with methotrexate $2.5 \mathrm{mg}$ every week into the proximal nail fold on each side of the nail [71]. Subungual hyperkeratosis and pitting had improved after six treatments.

One study has investigated the effect of combined oral and topical colloidal silicic acid on PP, psoriatic nail disease, and psoriatic arthropathy [72]. Patients were treated with oral colloidal silicic acid gel for 90 days, combined with topical silicic acid gel on psoriatic skin, but not on nail lesions. Of the 12 evaluable patients in the treated group, ten had psoriatic nail disease before treatment and five showed complete cure after treatment. The placebo-treated patients were not discussed in this publication.

Indigo naturalis, a dark-blue powder extracted from the leaves of indigo-bearing plants, is known as a dye in the Western World. In China it has been used orally for the treatment of many ailments, including psoriasis. In addition, topical use of indigo naturalis has shown to be effective in PP [73], but its use in nail psoriasis is limited by the recalcitrant and intense blue color. A refined formulation of indigo naturalis oil extract has a slight purplered color and is much more cosmetically acceptable. This compound $(0.1$ and $0.2 \mathrm{mg} / \mathrm{ml})$ is not yet commercially available but has been investigated in nail psoriasis in three studies [73-75]. In an open-label study, an improvement in NAPSI of $60 \%$, and in modified NAPSI of $68 \%$, was 
reported at 24 weeks after application of one drop onto the lateral nail folds, eponychium, and hyponychium [74]. After these first encouraging results, the same group published a randomized, observer-blind, vehicle-controlled, intrasubject trial that confirmed the previous results: at 12 weeks, the single-hand NAPSI had improved by $50 \%$, and the modified target NAPSI had improved by $59 \%$ [76]. No further improvement was seen between 12 and 24 weeks of treatment. Moreoever, subject global assessment (SGA) and physician global assessment (PGA) were improved in the indigo naturalis group than in the control group. In a third report, indigo naturalis extract was compared with topical calcipotriol solution [75]. Indigo naturalis extract showed significantly better results than calcipotriol for single-hand NAPSI at 24 weeks ( -51 vs. $-27 \%$, respectively; $p=0.007)$. Onycholysis and subungual hyperkeratosis improved most for both treatments. Irritation was the most common side effect of indigo naturalis extract; however, if these positive results can be confirmed by others, it could, in the future, become a genuine topical treatment option for nail psoriasis.

\section{Systemic Treatment Modalities}

Topical treatments for nail psoriasis have the obvious advantage of treating only the nail apparatus without exposing the rest of the body to the risk of adverse events. Therefore, topical treatments are generally the treatment of choice in patients with mild nail disease, unless the severity of concomitant PP or PsA creates a situation in which systemic therapy is preferable. Systemic treatments offer a valuable alternative in patients with severe nail disease with a major impact on QoL, pain, or on daily life and profession. Because psoriasis is a chronic disease, treatment must be continued for the long term, and even indefinitely. For this reason, long-term safety of any treatment should be considered and discussed in each patient. The choice for optimal systemic treatment in a patient does not only depend on the severity of the nail involvement but also on the presence or absence of psoriatic joint disease and skin disease, other diseases in the patient, the patient's preferences, long-term safety, experience of the prescriber, side effects, and costs of treatment. Methotrexate and apremilast are oral treatments that are effective in PP and PsA, while cyclosporine, fumaric acid, and retinoids are more effective in PP than PsA. The same consideration must be made in psoriasis patients who are going to be treated with biologics. While anti-TNF $\alpha$ and anti-IL-12/23 are effective in PsA, anti-IL-17 appears to be much more effective in skin involvement of the disease than in joint involvement.

\section{Oral Systemic Treatments}

\subsection{Methotrexate}

As an orally or subcutaneously administered systemic compound, methotrexate is one of the most used systemic treatments in PP, PsA, and nail psoriasis. It is cost effective and its efficacy in PP and PsA has been known for many years [59]. A wide range of potential side effects limit its use, including hepatotoxicity, ulcerative stomatitis, lymphopenia, nausea, low white blood cell count, and nausea. Studies focussing on methotrexate in nail psoriasis show efficacy, but significantly less than most biologics (Table 2) [77-80]. One RCT comparing methotrexate and cyclosporine in nail psoriasis showed a mean NAPSI improvement of $43 \%$ in methotrexate-treated patients and $37 \%$ in cyclosporine-treated patients at 24 weeks (not significant) [77]. The methotrexate group showed a significant improvement in nail matrix scores only, while the cyclosporine group showed significant improvement in nail bed scores only [77]. Methotrexate efficacy to treat nail psoriasis has also been compared with briakinumab in an RCT [78]. Target NAPSI improved $38 \%$ in methotrexatetreated patients and $56 \%$ in briakinumab-treated patients. Patients were treated with an extremely slowly increasing dose of methotrexate for 24 weeks, which might have had a negative impact on outcome. Smaller, non-controlled studies showed NAPSI improvements in the same range $[79,80]$.

\subsection{Cyclosporine}

The calcineurin inhibitor cyclosporine is a powerful immunosuppressive drug that, in dermatology, has a position in the treatment of several disorders, such as severe eczema, PP, and alopecia areata. Topical use of cyclosporine in nail psoriasis has been discussed in Sect. 5.6, but systemic use has also been the topic of several reports that, despite the lack of efficacy in PsA, show reasonable efficacy in the treatment of both nail bed and nail matrix signs of psoriasis [53, 79, 81-84]. One study indicated more efficacy of cyclosporine in nail bed psoriasis than in nail matrix psoriasis [77]. The efficacy of cyclosporine in comparison with other drugs has been reported in several studies. While Gumusel et al., who used the NAPSI as a scoring system, were unable to see significant differences between cyclosporine and methotrexate, most other studies used uncommon endpoints [77]. Mahrle et al. reported on a study of cyclosporine versus an obsolete retinoid — etretinate-using an unspecified 4-point scale [82]. Nail involvement had decreased by 46 and $34 \%$ compared with baseline after 22 weeks of treatment with cyclosporine and etretinate, 


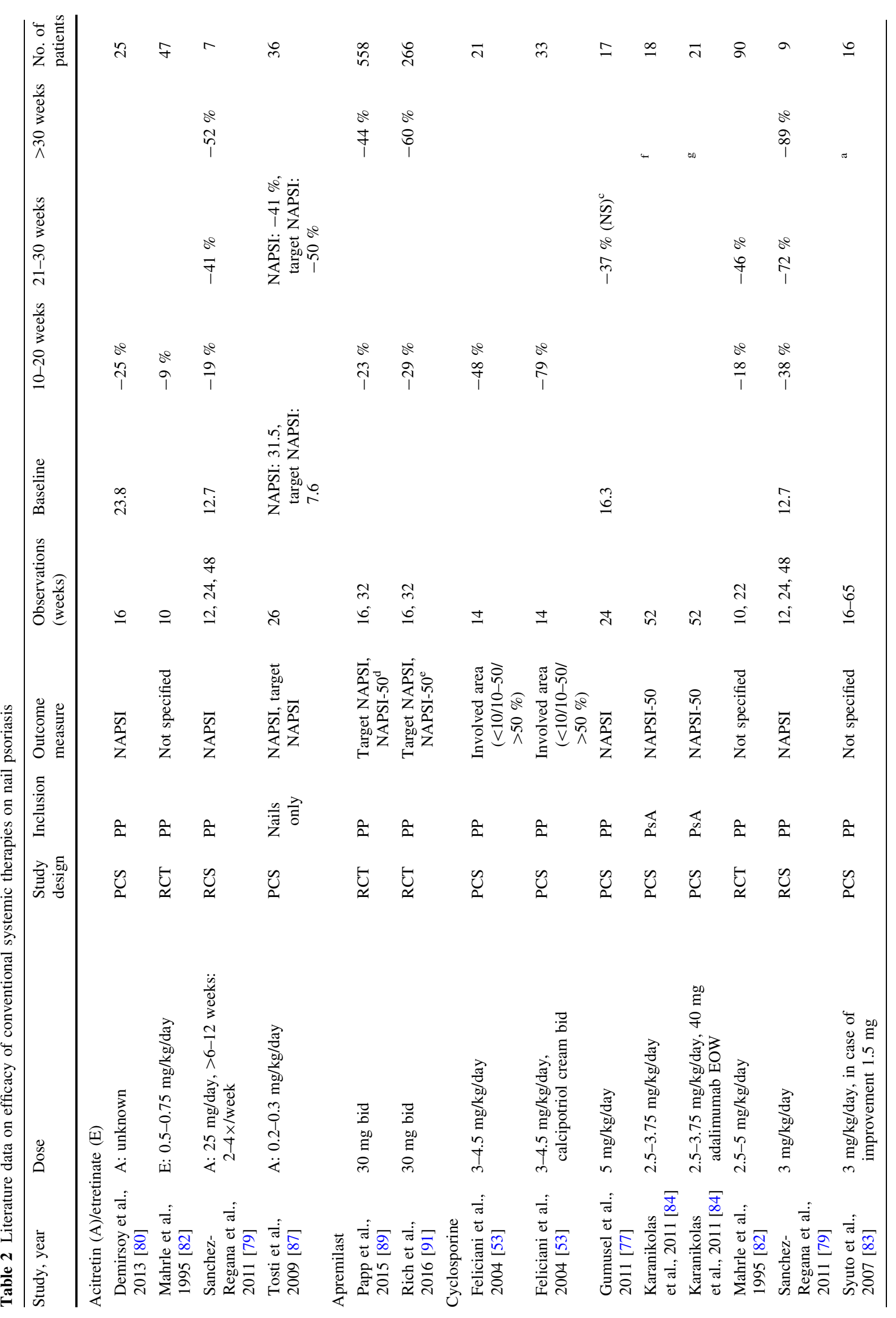




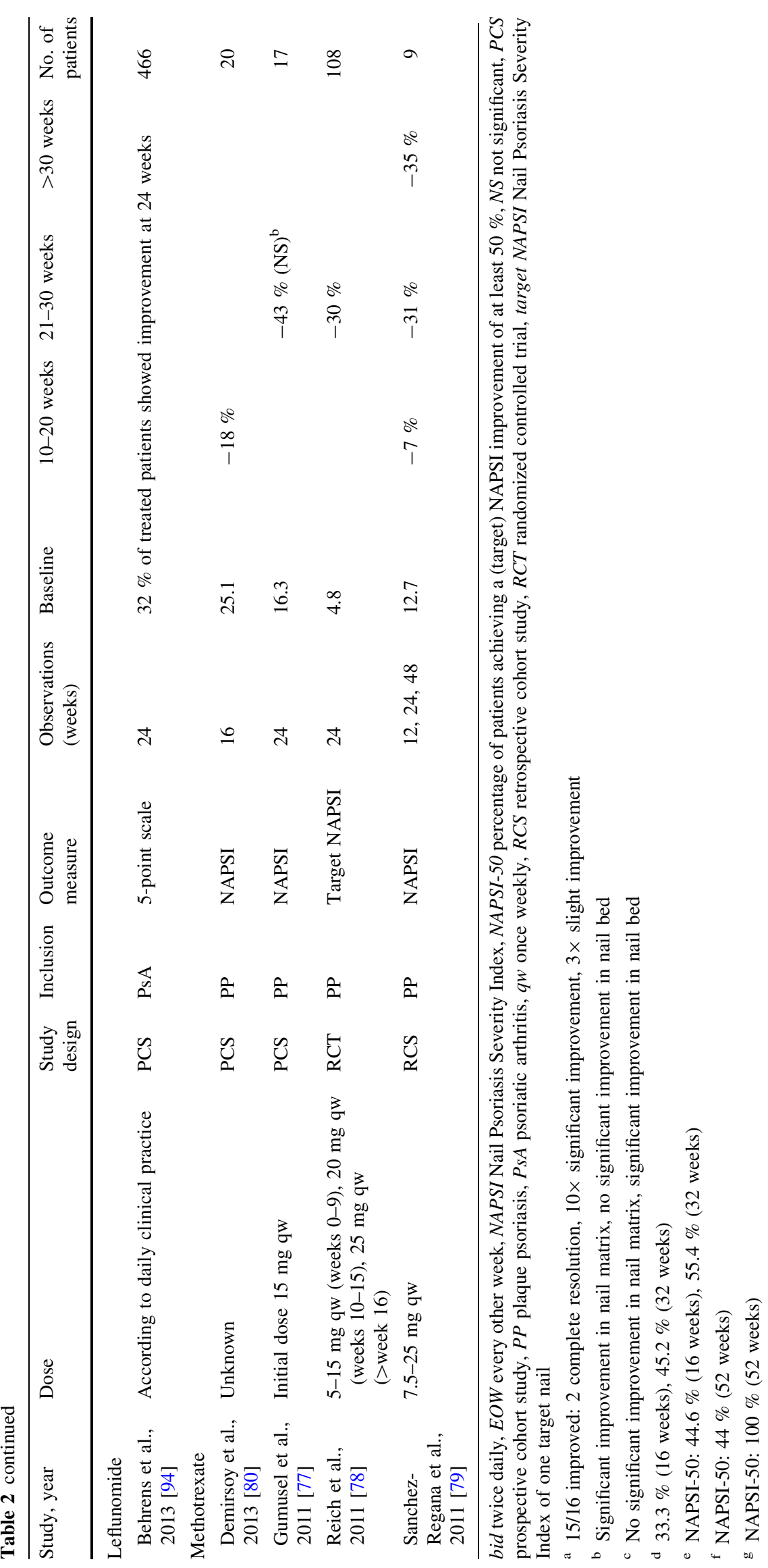


respectively. A 12-month comparison of cyclosporine with an anti-TNF $\alpha$ biologic_adalimumab-in PsA patients also suffering from nail psoriasis showed a NAPSI-50 in $44 \%$ of patients receiving cyclosporine, $56 \%$ of patients receiving adalimumab, and $100 \%$ of patients receiving a combination of the two drugs [84]. The finding that cyclosporine was comparable to biologics in treating nail psoriasis was also confirmed in a retrospective database study: cyclosporine was the most effective classical systemic treatment; NAPSI improved $89 \%$ at 48 weeks of treatment [79]. Concomitant use of cyclosporine and topical calcipotriol cream three times daily may further improve treatment results [53]. While most studies use doses between 3 and $5 \mathrm{mg} /$ day, one study from Japan investigated a lower dose of cyclosporine per day; the initial dose of $3 \mathrm{mg} / \mathrm{kg} /$ day was successfully reduced to $1.5 \mathrm{mg} / \mathrm{kg} /$ day in responders [83]. Cyclosporine use may be limited by the relatively high rates of side effects; the most serious adverse reactions are mostly reversible renal dysfunction and hypertension. Other side effects include fatigue, headache, paresthesia, hypertrichosis, gingival hyperplasia, and gastrointestinal disorders. After prolonged use, cyclosporine may play a role in the development of renal failure and several malignancies. For this reason, it is often considered as an effective treatment for severe cases of (nail) psoriasis, but only for a period of 6-12 months.

\subsection{Retinoids}

Acitretin is a non-immunosuppressing antipsoriatic drug that can be used for years in patients who can tolerate its side effects, such as cheilitis, dry mouth, and skin exfoliation [85]. Acitretin normalizes cellular differentiation and controls inflammation. The position of acitretin in the treatment of nail psoriasis is that of a rather slow-acting compound with moderate efficacy and action, particularly on nail bed signs of psoriasis (Table 2) [79, 80, 82, 86, 87]. Two open studies and one retrospective analysis indicated that a NAPSI improvement of $40-50 \%$ at 6-12 months may be expected [79, 87]. At earlier time points, acitretin is considerably less effective $[79,80]$. Acitretin can also be used as an adjuvant therapy to psoralen plus ultraviolet $\mathrm{A}$ (PUVA) and ultraviolet B (UVB), as discussed in Sect. 10.3.

\subsection{Apremilast}

Apremilast is an oral, small-molecule inhibitor of phosphodiesterase 4 that alters the expression of a variety of immune mediators [88]. It is USA FDA- and European Medicines Agency (EMA)-approved for the treatment of adults with psoriasis and PsA. Two RCTs (ESTEEM 1 and 2) in which nail psoriasis was a secondary endpoint investigated its efficacy in nail psoriasis (Table 2) [89-91]. At 16 weeks, target NAPSI improvement was limited, but after 32 weeks of apremilast, NAPSI had improved 43.6 and $60.0 \%$ in ESTEEM 1 and ESTEEM 2, respectively. Both nail matrix and nail bed psoriasis improved significantly. The percentage of patients achieving NAPSI-50 at 32 weeks in ESTEEM 1 and ESTEEM 2 was 45.2 and $55.4 \%$, respectively. The very liberal definition of nail psoriasis in these studies (NAPSI $\geq 1$ ) makes it hard to draw conclusions about efficacy in clinically relevant nail psoriasis. Because apremilast is generally well tolerated and has no need for biochemical follow-up it may be an attractive choice for patients in who systemic treatments are indicated. The very high costs of treatment may limit its use in many patients.

\subsection{Miscellaneous Systemic Therapies: Fumaric Acid Esters, Sulfasalazine, and Leflunomide}

Fumaric acid esters (FAEs), sulfasalazine, and leflunomide have also been reported to be effective in nail psoriasis; however, based on the current knowledge, they cannot be advised for this indication. FAEs have been used for the treatment of chronic PP since 1959. The commercially available form, containing mainly dimethylfumarate, is currently a first-line systemic therapy for severe chronic PP in several European countries; it is not effective in PsA. The general opinion is that it has little efficacy in nail psoriasis, but one case has been reported in which it was effective both on nail bed and nail matrix psoriasis [92]. Side effects are frequent in patients using FAEs, such as episodes of flushing, abdominal pain, and a decrease in lymphocyte count.

Sulfasalazine is an aminosalicylate used as a traditional disease-modifying antirheumatic drug (DMARD) for the treatment of peripheral involvement in PsA. Sulfasalazine is metabolized by gut flora into sulfapyridine and 5-aminosalicylic acid, which act as anti-inflammatory agents. The role of sulfasalazine in cutaneous psoriatic lesions and nail psoriasis is not generally accepted. One case has been reported on the use of sulfasalazine in the treatment of psoriatic nail lesions [93]. After 3 months, nail lesions started to recede and disappeared progressively. No other cases have been reported in literature.

Leflunomide is another DMARD used to treat PsA. It is a de novo pyrimidine biosynthesis inhibitor with immunomodulatory properties. One prospective study assessed the clinical effectiveness and safety of leflunomide in patients with PsA, and also in psoriatic nail disease [94]. Unlike most studies on PP, patients in this study also received concomitant antiinflammatory/antirheumatic drugs, systemic corticosteroids, and other DMARDs, predominately methotrexate. Significant improvements were 
experienced in $32 \%$ of patients with nail lesions. The most frequent adverse events were diarrhea, alopecia, hypertension, and pruritus.

\section{Biologics}

The introduction of biologics has led to a major step forward in the treatment of severe PP and PsA, and may have the potential to revolutionize the management of patients with disabling psoriatic nail disease. Anti-TNF $\alpha$ treatments were the first, very effective biologics that became available to psoriasis patients. More recently, other mechanisms of the psoriatic inflammatory reaction can be suppressed with anti-IL-17, and anti-IL-12/23 monoclonal antibodies. Consequently, the therapeutic armamentarium is expanding for patients with moderate to severe PP or PsA who are unresponsive to conventional systemic therapies or have contraindications for these therapies. Infliximab, etanercept, and adalimumab are anti-TNF $\alpha$ treatments available both for PP and PsA patients, while golimumab and certolizumab pegol only have registration for PsA. The biologic ustekinumab, which targets IL-12/23, and the anti-IL17 antibody secukinumab are also available for both PP and PsA. All these biologics appear to have a positive effect on nail psoriasis, without major differences in efficacy between the populations they are investigated in, i.e. PP or PsA (Table 3). In general, nail responses are slow but continue to improve, lagging behind cutaneous responses over time. While the onset of response in nail psoriasis is mostly noticeable after approximately 12 weeks, further improvement or even complete clearance can be seen through to 1 year of treatment. Nonetheless, nail, skin, and joint manifestations of psoriasis generally improved in parallel based on correlation between NAPSI, Psoriasis Area Severity Index (PASI), and American College of Rheumatology (ACR) responses over time. Overall, patients with greater skin or joint responses also demonstrated better nail responses. On the other hand, the presence of nail disease was not shown to predict good response of the arthritis [95], and improvement of nail psoriasis by a biologic did not depend on the presence or absence of PsA [96].

\subsection{Inhibition of Tumor Necrosis Factor- $\alpha$}

$\mathrm{TNF} \alpha$ is a proinflammatory cytokine that plays a major role in psoriasis by promoting an inflammatory infiltrate into the skin and inducing keratinocyte proliferation and preventing keratinocyte apoptosis, which directly contributes to the characteristic skin and nail lesions. Anti-TNFa has shown to be a relatively safe and very effective treatment for PP, PsA, and nail psoriasis (Table 3). The originally high cost of these treatments has limited their use in nail psoriasis, but since lower-cost biosimilars for infliximab and etanercept have been approved in the US and/or the EU they may become available to more patients with nail psoriasis. Infliximab, etanercept, adalimumab, golimumab, and certolizumab pegol all appear to be effective treatments for nail psoriasis and will be discussed individually in the following sections. The most often reported adverse effects related to the use of TNF $\alpha$ antagonists are activation of opportune infections, including mycobacterium tuberculosis (TB), demyelinating diseases, congestive heart failure, induction of the formation of autologous antibodies, and antibodies neutralizing anti-TNF $\alpha$ drugs [97]. The development of anti-TNF $\alpha$-induced lupus or classical druginduced lupus is more rarely reported. Contraindications for the use of anti-TNF $\alpha$ medications are related to the adverse events, i.e. chronic infection, such as active TB or other severe infections, moderate to severe heart failure, as well as pregnancy and nursing. TB should be ruled out before starting therapy. Patients with hematological changes, demyelination processes, and recent neoplasms should not be treated, or should be treated only in close collaboration with other relevant specialists.

\subsubsection{Infliximab}

Infliximab was approved in the US in 1998 for the treatment of Crohn's disease. Thereafter, infliximab has had its indication expanded, and in 2005/2006 became available for the treatment of PsA and PP. It is a chimeric anti-TNFa immunoglobulin (Ig) G1 monoclonal antibody that is comprised of human antibody constant regions and murine variable regions. Infliximab binds both soluble and membrane-bound TNF $\alpha$, preventing it from binding its receptor, resulting in a decrease in epidermal T-cell infiltration. The chimeric character of infliximab may result in a higher formation of neutralizing antibodies than occurs with fully human(-ized) antibodies. A concern related to the formation of these antibodies is the decreased efficacy of infliximab over time, which may require increased infusion frequencies, higher doses to maintain a clinical response and disease control, and infusion reactions, occurring in $16 \%$ of infliximab-treated patients [98]. These reactions can involve chills, fever, headache, flushing and urticaria, myalgia and arthralgia, nausea, dyspnoea, and hypotension [99-101]. Most infusion-related reactions are mild and can usually be ameliorated by reducing the rate of infusion rather than discontinuing therapy. However, anaphylactic reactions are also possible, requiring surveillance during and for $1 \mathrm{~h}$ after infusion. A type IV delayed hypersensitivity reaction may also present 3-12 days after infusion and produce a serum sickness-like reaction [102]. A study comparing the incidence of onychomycosis inpatients 


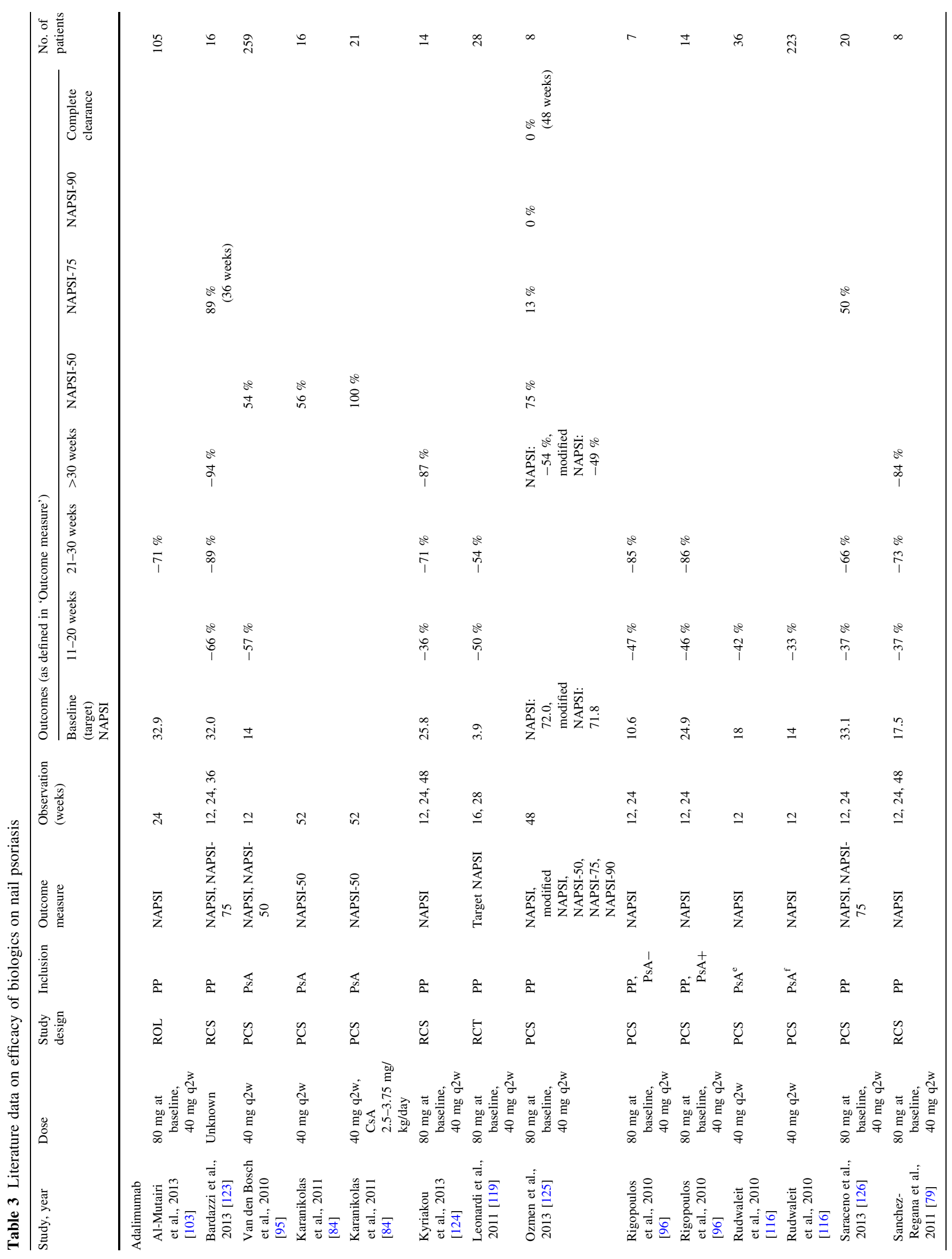




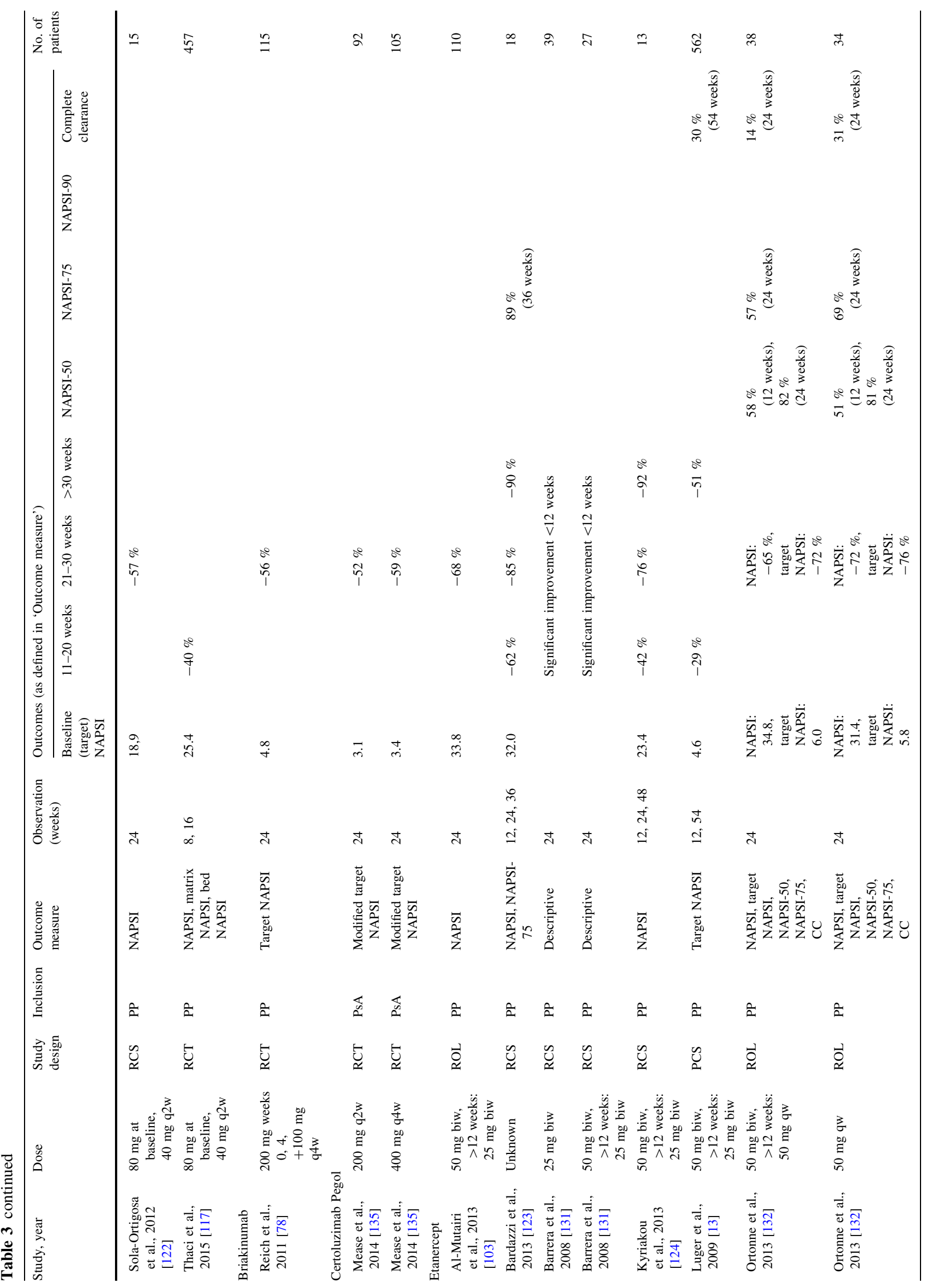




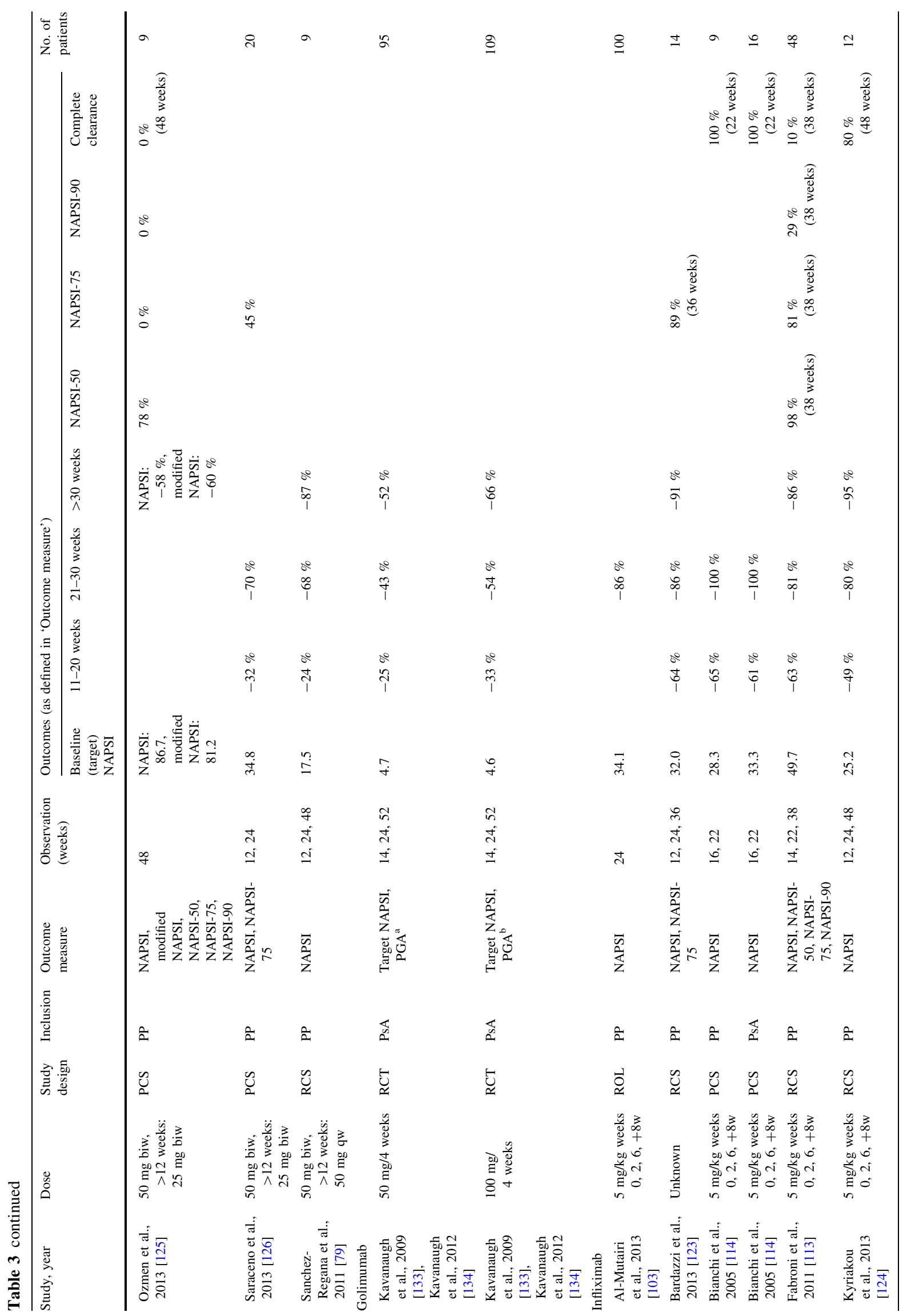




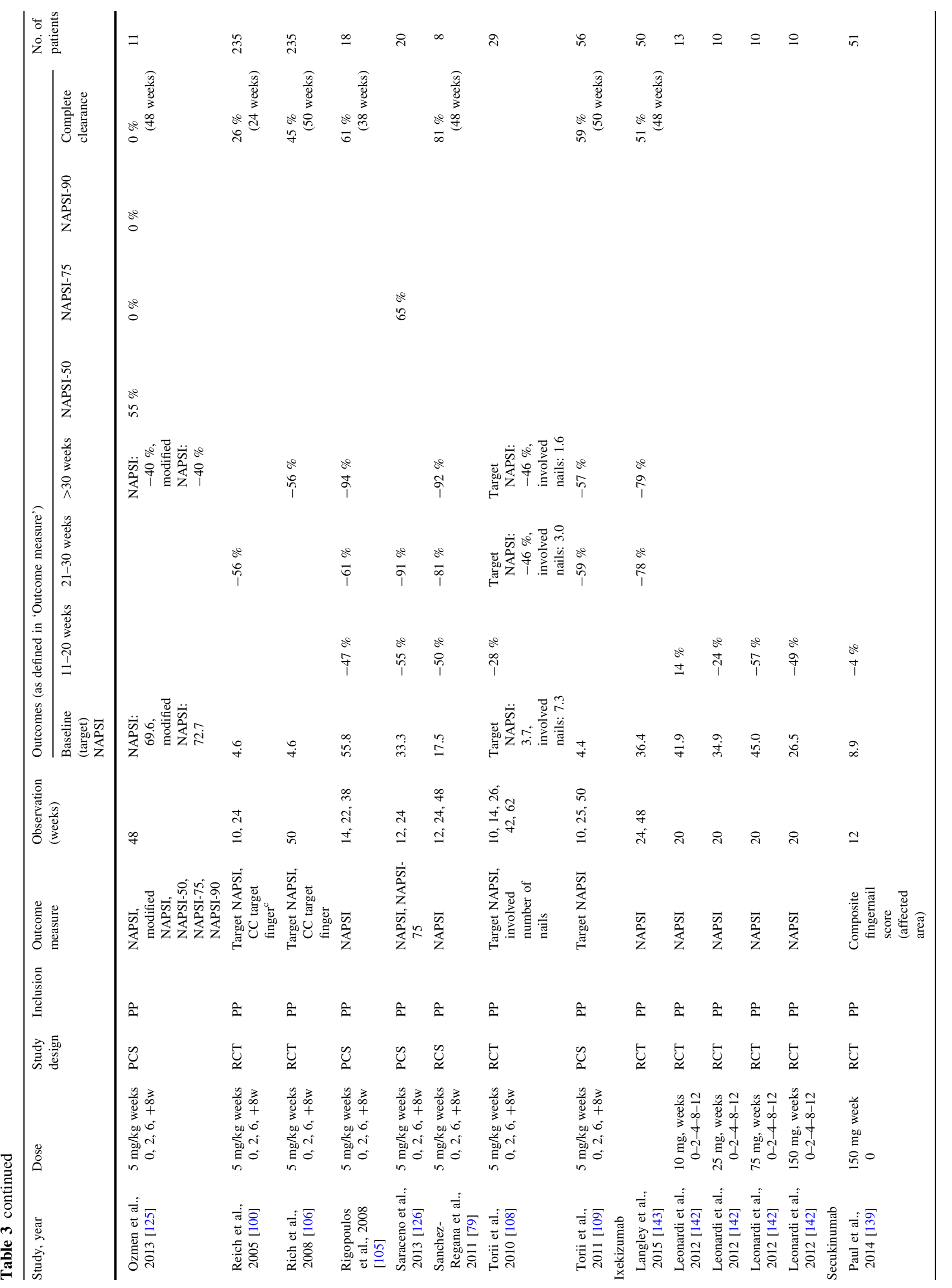


Nail Psoriasis: A Review of Treatment Options

693

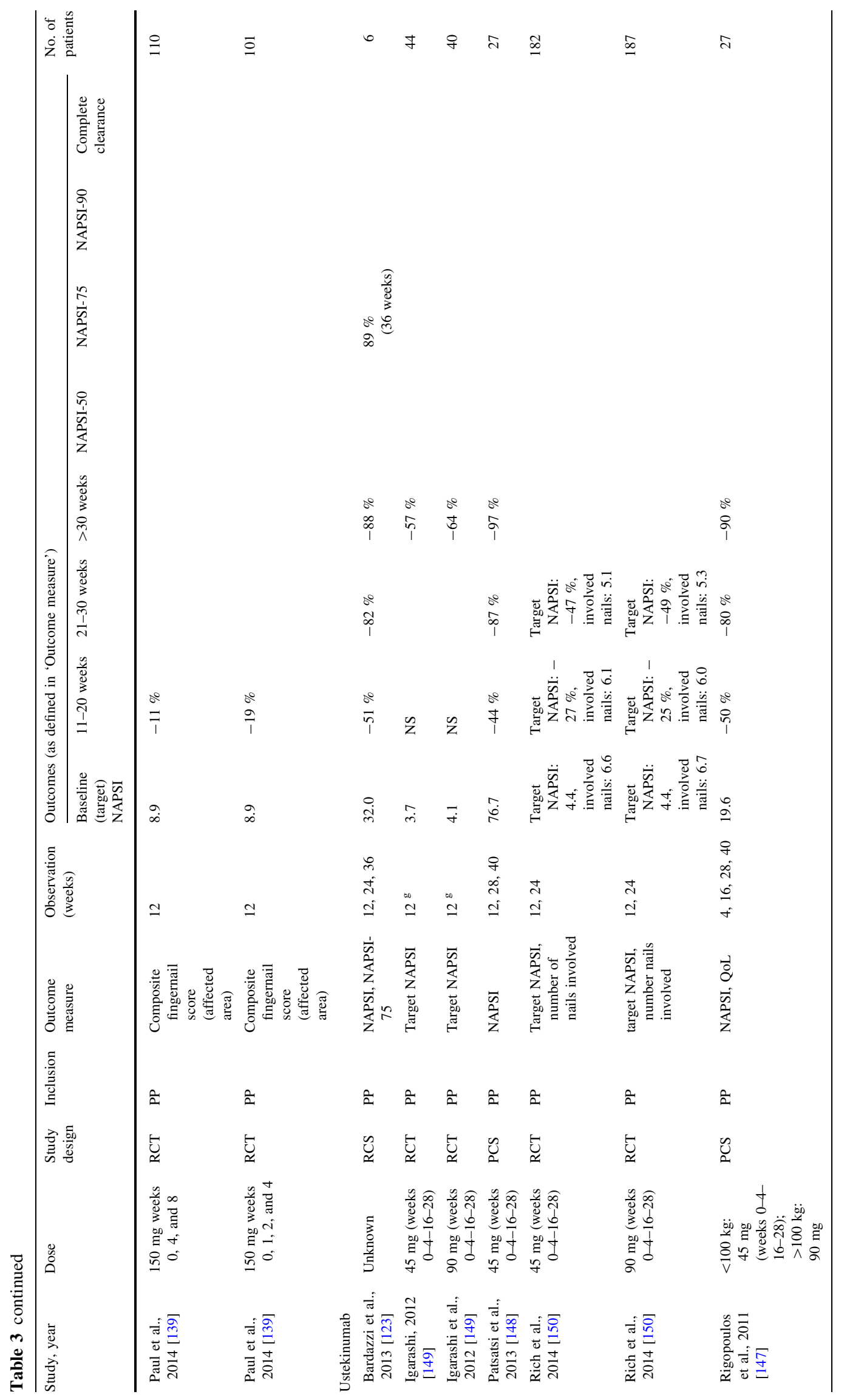

$\triangle$ Ads 


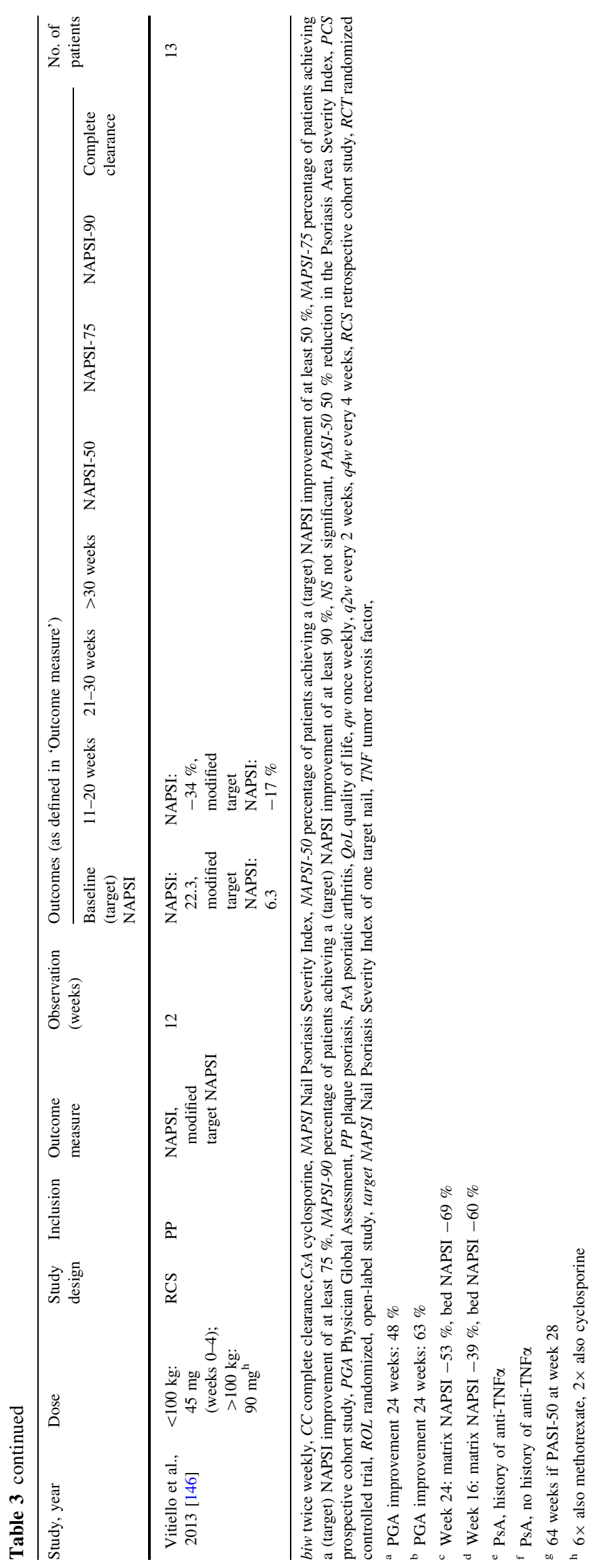


treated with infliximab, etanercept, and adalimumab has shown a statistical positive association between fungal infections of the nail in psoriasis patients and the use of infliximab [103]. Thirty-three percent of patients receiving infliximab had a positive fungal culture, while in patients receiving etanercept and adalimumab, as well as controls, this percentage was $15.5,13.3$, and $13.9 \%$, respectively.

Several studies have proven the beneficial effect of infliximab on nail psoriasis, both in PsA and PP patients, and also in patients with severe nail psoriasis at baseline (Table 3). No differences between efficacy in nail bed and nail matrix psoriasis have been reported. Patients with a high PASI response have a more rapid and profound nail response than patients with a poorer PASI response [104]. In addition, QoL improves during infliximab treatment in nail psoriasis [105].

The first report on infliximab in nail psoriasis came from the EXPRESS study. Reich et al. reported on 235 psoriatic patients who also had nail involvement [100] and who were further analyzed by Rich et al. [106]. Patients were treated with infliximab or placebo up to week 46. Complete clearance of a target finger was seen in 26 and $45 \%$ of patients at week 24 and week 50, respectively, while at both time points the mean improvement in target NAPSI was $56 \%$. At week 24, the mean percentage improvements in nail matrix and nail bed features were 52.9 and $69.2 \%$, respectively. Improvement of both nail bed and nail matrix psoriasis was also reported from Japan [107]. These authors also reported a randomized, double-blind, placebocontrolled multicenter trial [108]. Improvements in target NAPSI at weeks 26-66 was $46 \%$, and the number of affected nails had decreased by $78 \%$ at week 66. An openlabel, uncontrolled study resulted in a target NAPSI improvement of $59 \%$ at week 26, and $57 \%$ improvement at week 50 [109]. Other case reports and open-label studies reported even more improvement in severity of nail psoriasis [105, 110-112]. A retrospective study of 48 patients in a group of patients with severe nail psoriasis (NAPSI at baseline, 49.7) also showed excellent real-life results [113]. At 22 weeks, NAPSI had improved to $80.8 \%$, and at 38 weeks had improved to $85.9 \%$. Almost all patients achieved NAPSI-50, $81 \%$ achieved NAPSI-75, and $29 \%$ achieved NAPSI-90. Complete clearance was seen in $10 \%$ of patients.

Infliximab appears to be equally effective for the treatment of nail psoriasis in patients with PsA and PP. Bianchi et al. reported on nine patients with PP and 16 patients with arthropathic psoriasis who were treated for 16 weeks in an open-label, non-controlled, prospective study [114]. At week 16, NAPSI improvement in PP patients was $64.7 \%$, and $60.7 \%$ in PsA patients.

Prospective and retrospective studies comparing several systemic and biologic therapies have been conducted and will be discussed in Sect. 9. The efficacy of infliximab in these trials is in the same range as in the above-mentioned studies.

Considering the results of these studies, one can conclude that while nail response generally lags behind cutaneous response in these patients, similar excellent degrees of response can be attained following 6-12 months of infliximab treatment. Comparing data from trials on all discussed biologics, infliximab might be the fastest-acting treatment for nail psoriasis.

\subsubsection{Adalimumab}

PP and PsA are both indications for adalimumab, a fully human anti-TNF $\alpha$ IgG1 monoclonal antibody. On binding $\mathrm{TNF} \alpha$, adalimumab neutralizes the biological activities of this cytokine by blocking its interaction with the p55 and p75 cell surface TNF receptors, and modulating biological responses that are induced or regulated by $\mathrm{TNF} \alpha$. Initially, its efficacy in nail psoriasis was claimed in a case report [115] but clinical studies have followed (Table 3). The first study was an open-label study in patients with mild to moderate nail psoriasis [96]. After 24 weeks of treatment, the fingernail NAPSI had improved $85 \%$. No differences in efficacy were seen between patients with PP only and patients with both PP and PsA. Another open-label study investigated the efficacy of adalimumab in 259 PsA patients only [116]. At week 12, NAPSI improvement in patients naive for anti-TNFa $(-33 \%)$ was not significantly different from patients with prior treatment with infliximab and/or etanercept $(-42 \%)$. In the STEREO study, another prospective, open-label, uncontrolled study that evaluated the effectiveness of adalimumab in patients with active PsA, the median reduction in NAPSI score was $57 \%$ in 259 patients with both active arthritis and nail psoriasis at 12 weeks [95]. NAPSI-50 was recorded in $54 \%$ of patients with a baseline NAPSI score of at least 10 . This study also searched for predictors of good clinical responses for arthritis, skin, and nail lesions. Nail disease turned out to be unable to predict good response of the arthritis.

The BELIEVE study and the REACH study are two randomized, double-blind, controlled studies in PP patients reporting on the efficacy of adalimumab in nail psoriasis [117-121]. A subgroup analysis of the BELIEVE study of 457 patients also with nail psoriasis claimed a reduction in the NAPSI nail matrix component of $39 \%$ and NAPSI nail bed component of $60 \%$ at week 16 [117]. These results appear to contradict the overall NAPSI reduction of $40 \%$ at week 16 because both the matrix and bed signs are equally important in the calculation of the overall NAPSI. Another subgroup analysis of the BELIEVE study focussed on the influence of PsA on the efficacy of adalimumab [120]. Numerically larger decreases in mean NAPSI scores 
were observed over time for patients without a history of PsA compared with patients with a history of PsA; however, the differences between groups were not statistically significant. Efficacy on nail psoriasis has been a secondary endpoint in the REACH study investigating adalimumab for the treatment of moderate to severe chronic PP of the hands and feet [119]. Target NAPSI in 28 patients with nail psoriasis had improved 50 and $54 \%$ at weeks 16 and 28, respectively. In a post hoc analysis of the REACH study among 31 patients with nail psoriasis, a greater percentage of adalimumab-treated patients achieved NAPSI-50 $(56.5 \%)$ versus placebo $(12.5 \%)$ at week 16 [121]. A remarkable finding in this study was that NAPSI-50 responders experienced a greater mean reduction in pain visual analog scale (VAS) score $(86.1 \%)$ than NAPSI-50 non-nesponders $(1.4 \%)$ among patients treated with adalimumab.

Daily practice studies were published by Sola-Ortigosa et al. [122], Bardazzi et al. [123], and Kyriakou et al. [124]. Sola-Ortigosa measured NAPSI improvement of $57 \%$ in 15 patients after 24 weeks of adalimumab treatment, while a better NAPSI improvement ( $89 \%$ ) at this time point was shown by Bardazzi et al. in 16 patients receiving adalimumab, increasing to $94 \%$ at 36 weeks of follow-up. In the study by Kyriakou et al., the 14 patients receiving adalimumab had a NAPSI improvement of $71 \%$ at week 24 and $87 \%$ at week 48 .

Prospective and retrospective studies comparing several systemic and biologic therapies have been conducted and will be discussed in Sect. 9. The efficacy of adalimumab in these trials is in the same range as in the above-mentioned studies [79, 84, 125, 126]. Briefly, Ozmen et al. [125] reported a reduction in NAPSI of $54 \%$ in eight patients receiving adalimumab at 48 weeks, Saraceno et al. [126] reported a NAPSI reduction of $66 \%$ in 20 adalimumabtreated patients, and in the eight patients reported by Sanchez-Regana et al. [79], the percentage of reduction in the NAPSI score was 37, 73, and $84 \%$ at 12,24 , and 48 weeks, respectively. Finally, after 12 months of treatment, Karanikolas et al. [84] reported NAPSI-50 in $56 \%$ of patients receiving adalimumab, $44 \%$ of patients receiving cyclosporine, and $100 \%$ of patients receiving a combination of the two drugs. Summarizing these results, it is clear that adalimumab is an effective treatment for psoriasis of the nail bed and the nail plate, both in PP patients and in PsA patients. The most commonly reported side effects of adalimumab are comparable with those of other biologics. A prospective study was unable to see an increased incidence of onychomycosis in psoriasis patients treated with adalimumab for 24 weeks [103].

\subsubsection{Etanercept}

Etanercept is a fusion protein of the TNF receptor and Fc end of the IgG1 antibody, which binds with and antagonizes the action of TNF $\alpha$. It is approved for the treatment of rheumatoid arthritis, juvenile rheumatoid arthritis, ankylosing spondylitis, PsA, and PP. Its efficacy in PP has been shown in many clinical trials since 2003 [127] but its usefulness in nail psoriasis started to be reported several years later in case reports [128-130]. Another publication claiming the clinical efficacy of etanercept in nail psoriasis was published in 2008, and reports from a retrospective and observational study in PP patients [131]. No data on nail psoriasis were given, but a significant improvement of nail involvement was noticed within 12 weeks. In 2009, a post hoc analyses of the CRYSTEL study was published [13]. At 12 weeks of etanercept treatment, target NAPSI had improved $29 \%$. At 54 weeks, at end of follow-up, target NAPSI improvement had increased to $51 \%$. Patients with nail psoriasis also showed significant and clinically meaningful improvement with etanercept therapy in the Dermatology Quality of Life Index (DLQI) and EQ-5D VAS, QoL measures for which they had worse scores than psoriasis patients without nail involvement at baseline $(p<0.001)$. A clinically meaningful improvement in QoL was also shown in the NAIL study, a 24-week randomized clinical trial investigating the efficacy and safety of two doses of etanercept in which improvement of nail psoriasis was the primary endpoint [132]. This study was unable to show statistical differences between etanercept $50 \mathrm{mg}$ twice weekly for 12 weeks followed by $50 \mathrm{mg}$ once weekly for another 12 weeks (Group A), and etanercept $50 \mathrm{mg}$ once weekly for 24 weeks (Group B). Target NAPSI in Group A had improved $72 \%$ and target NAPSI had improved $76 \%$ in Group B. In addition, overall NAPSI showed an important improvement (see Table 3). Daily practice studies were published by several other authors [122-124]. Bardazzi et al. studied 18 patients receiving etanercept, and showed a NAPSI response of $85 \%$ at 24 weeks of treatment, and $94 \%$ NAPSI improvement at 36 weeks of follow-up. The 13 patients receiving etanercept in the study by Kyriakou et al. had a NAPSI improvement of $76 \%$ at week 24, and $92 \%$ at week 48 .

Other studies of etanercept in nail psoriasis are comparative studies that will be discussed in Sect. 9. These studies report improvement of nail psoriasis in the same range as the above-mentioned studies. Briefly, SanchezRegana et al. treated nine patients with etanercept [79], and reduction in the NAPSI score was $24 \%$ at week 12, increasing to $87 \%$ at 48 weeks. Ozmen et al. reported a 
$57 \%$ NAPSI in nine patients after 48 weeks of etanercept treatment reduction [125], and Saraceno et al. measured NAPSI improvement of $70 \%$ in etanercept-treated patients [126]. The most commonly reported side effects of adalimumab are comparable with those of other biologics, but antibody formation and its consequences are claimed to occur less frequently. A prospective study was unable to see an increased incidence of onychomycosis in psoriasis patients treated with etanercept for 24 weeks [103].

\subsubsection{Golimumab}

Golimumab is a human monoclonal anti-TNFa antibody that has a registration for the treatment of PsA but not for PP. In an RCT in PsA patients-the GO-REVEAL studynail involvement was a secondary endpoint (Table 3) [133]. Approximately half of the patients were also taking methotrexate. In patients treated with the common dose of golimumab $50 \mathrm{mg}$ every 4 weeks, improvement was reported both in target NAPSI ( $-43 \%)$, and PGA (-48 \%) at 24 weeks. Target NAPSI improvement was even slightly higher $(-52 \%)$ at 52 weeks [134].

\subsubsection{Certolizumab Pegol}

Certolizumab pegol is a humanized mouse monoclonal antibody to TNF $\alpha$ that is chemically modified by PEGylation, resulting in a final product that has a prolonged halflife in patients. In the maintenance phase of treatment, patients receive one dose every $2-4$ weeks, which is slightly less frequent than with other anti-TNF $\alpha$ antibodies. It is registered for PsA patients but several trials in PP patients are ongoing. The RAPID-PsA study was an RCT investigating certolizumab pegol in PsA patients in which psoriatic nail involvement was a secondary endpoint [135]. As in many studies primarily focussing on PsA, concomitant use of methotrexate, sulfasalazine, leflunomide, or oral corticosteroids was permitted. Mean target NAPSI change from baseline at week 24 was $-52 \%$ with certolizumab pegol $200 \mathrm{mg}$ every 2 weeks and $-59 \%$ with certolizumab pegol $400 \mathrm{mg}$ every 4 weeks versus $-32 \%$ with placebo ( $p=0.003$ and $p<0.001$, respectively) [Table 3]. Common side effects are nasopharyngitis, upper respiratory tract infection, headache, and pruritus.

\subsection{Anti-Interleukin (IL)-17 Treatments}

Fundamental immunological research pointed to a central pathogenic pathway in which IL-17A is the most critical T-cell-derived cytokine in altering skin function, while, in turn, IL-17-producing T cells (T17 cells) are regulated by IL-23. There is thus an intimate link between dissection of pathogenic pathways for psoriasis and new emerging therapies for this disease [136]. Several clinical trials in PP have suggested this concept being correct (Table 3) [137139], but others have criticized some of these trials [140, 141]. Two IL-17 neutralizing agents (i.e. secukinumab and ixekizumab) have been investigated in nail psoriasis. Common adverse effects of anti-IL-17 treatments are nasopharyngitis, upper respiratory tract infection, and headache. Secukinumab may exacerbate Crohn's disease, and anaphylaxis and cases of urticaria are reported. These drugs should be used with caution in patients with chronic infection or a history of recurrent infection, and all patients should be evaluated for TB before initiating.

\subsubsection{Secukinumab}

Secukinumab is a human monoclonal $\mathrm{IgG} 1 \mathrm{k}$ antibody that targets IL-17A and has been approved for the treatment of PP and PsA. Little is known about the efficacy of secukinumab in nail psoriasis. One subanalysis of a dose-finding RCT reported secukinumab improvement of nail lesions in moderate to severe PP [139]. Unfortunately, the NAPSI scale was not used but rather a never before described composite score (range 0-20) was used, and no patients were treated using the eventually approved dose for PP. From this study, no conclusions on efficacy in nail psoriasis can be drawn; patients treated with secukinumab $150 \mathrm{mg}$ at baseline and weeks 1,2, and 4 had an improvement of $19 \%$ in the composite fingernail score. In patients treated with $150 \mathrm{mg}$ at baseline and weeks 4 and 8 , this improvement was $11 \%$ (Table 3). A multicenter RCT investigating the efficacy of subcutaneous secukinumab 150 and $300 \mathrm{mg}$ at 16 weeks in subjects with moderate to severe nail psoriasis has recently been conducted but the results have not yet been published in a peer-reviewed journal. This TRANSFIGURE study also assesses safety, tolerability and long-term efficacy up to 132 weeks.

\subsubsection{Ixekizumab}

Ixekizumab is a humanized IgG4 monoclonal antibody neutralizing IL-17A intended to treat PP. Although this drug does not yet have a registration, some data are available suggesting efficacy in nail psoriasis (Table 3) [142]. Significant reductions in NAPSI scores were observed as early as 2 weeks, and these effects were sustained through 20 weeks. At 12 weeks, NAPSI had decreased significantly $(-57 \%)$ in patients treated with ixekizumab $75 \mathrm{mg}$ at weeks $0,2,4,8$, and 12 . The same patients have also been reported in an open-label extension receiving $120 \mathrm{mg}$ of ixekizumab subcutaneously every 4 weeks from week 20, however only in patients not achieving a PASI 75 [143]. In these patients, mean NAPSI had improved $78 \%$ at week 24 of the extension (week 44 
of the trial), and $79 \%$ at week 48 of the extension (week 68 of the trial). Complete clearance of the nails was achieved in a high proportions of patients: $43 \%$ at week 44, and $51 \%$ at week 68. Common side effects are nasopharyngitis, upper respiratory tract infection, headache, and injection site erythema.

\subsection{Anti-IL-12/23 Treatments}

Ustekinumab is the only anti-IL-12/23 treatment currently available for the treatment of PP and PsA. This antibody binds with high specificity and affinity to the shared p40 protein subunit of cytokines IL-12 and IL-23, blocking the differentiation and expansion of Th1 and Th17 populations. Briakinumab is another human monoclonal antibody targeting IL-12/23 being developed for the treatment of rheumatoid arthritis, inflammatory bowel disease, and multiple sclerosis. Further development for psoriasis has been discontinued, possibly because of cardiovascular adverse events [144]. Clinical efficacy of briakimumab in nail psoriasis has been shown in an RCT in which it was compared with methotrexate (Table 3) [78]. At 24 weeks, target NAPSI improved $56 \%$ in briakinumab-treated patients, and $38 \%$ in methotrexate-treated patients.

\subsubsection{Ustekinumab}

Ustekinumab is a human anti-IL-12/23 IgG1 monoclonal antibody that is indicated for the treatment of moderate to severe PP and for PsA, while early case reports also suggested good efficacy on nail involvement in psoriasis [145]. In a series of 13 cases, a reduction in overall NAPSI of $34 \%$ and modified target NAPSI of $17 \%$ was reported as early as week 12 (Table 3) [146]. The first prospective study focussing on nail improvement by ustekinumab was an open, unblinded study in a population treated with ustekinumab for cutaneous psoriasis [147]. Statistically significant improvement was present from week 4 (NAPSI $-13 \%)$ and was relevant at weeks $16(-50 \%), 38$ $(-80 \%)$, and $40(-90 \%)$. Similarly, QoL scores significantly improved at all time points. Another open-label, uncontrolled study of ustekinumab in nail psoriasis confirmed these excellent results [148]. Patients were treated for 40 weeks, resulting in a reduction in mean NAPSI of 87 and $97 \%$ at weeks 28 and 40, respectively. Two randomized, placebo-controlled trials were also able to report efficacy of ustekinumab in nail psoriasis but at a slightly lower range [149, 150]. The PHOENIX-1 study was a randomized, placebo-controlled, crossover study in 766 psoriasis patients who were treated with ustekinumab $45 \mathrm{mg}$ (or $90 \mathrm{mg}$ if $>100 \mathrm{~kg}$ ) in a normal regimen [150]. Over $70 \%$ of patients had nail psoriasis. An interesting but not surprising finding was that nail improvements were higher in patients with a good PASI response; improvement in NAPSI ranged from $30 \%($ PASI $<50)$ to $57 \%$ $($ PASI $\geq 90)$ at 24 weeks. Compared with baseline, the proportion of ustekinumab-treated patients with pitting decreased by approximately $20 \%$, and the proportion with onycholysis decreased by over $30 \%$ in both groups, indicating positive effects of ustekinumab, both on nail bed and nail matrix psoriasis. Substantial improvements in nail PGA scores were also observed, with the majority of patients with a baseline nail PGA score of $\geq 3$ (moderate) achieving improvement by at least 1 point. Igarashi et al. investigated the safety and efficacy of normal- and highdose ustekinumab in 102 Japanese patients with moderateto-severe plaque-type psoriasis [149]. At week 12, no significant NAPSI improvement compared with placebo was measured. At week 64, these investigators reported a mean improvement in target NAPSI scores of 57 and $68 \%$ for the ustekinumab 45 and $90 \mathrm{mg}$ groups, respectively. Only patients with at least a PASI-50 improvement were treated longer than 28 weeks. Because improvement of NAPSI often follows improvement in PASI, as has been shown in the PHOENIX-1 study, the reported percentages of nail improvement at 64 weeks may be an overestimation.

Ustekinumab studies show nail responses similar to the responses with other biologics. Comparative studies are discussed in Sect. 9, but a small retrospective study was unable to show differences between clinical efficacy on nail psoriasis of ustekinumab compared with several antiTNF $\alpha$ treatments [123]. Contraindications and adverse events of ustekinumab are comparable with anti-TNFa treatments. Patients should be evaluated for TB infection prior to administration, and any active serious infection should be treated before starting therapy. Ustekinumab may increase the risk of infections and reactivation of latent infections; serious bacterial, fungal, and viral infections. It may also increase the risk of malignancy, and all patients need to be monitored for non-melanoma skin cancer.

\section{Comparative Studies with Systemic Therapies}

As discussed above, biologics and conventional systemic treatments have been shown, in numerous studies, to be potent suppressors of psoriatic nail disease. The degree of improvement of clinical signs attributed to individual treatments is very different, even between different studies of the same drug. This makes it virtually impossible to compare results of distinct studies with different drugs. The explanations for these differences are manifold. The 'NAPSI' scoring systems used are extremely heterogeneous, the primary inclusion criterion for a study can be 
PP, PsA, or nail psoriasis, the quality of the studies differs, and some studies use very liberal criteria to define nail psoriasis, while others included only patients with severe psoriasis. Finally, the period of follow-up is of the utmost importance in the judgment of the results because further improvement may occur up to 1 year. These reasons underline the need for studies in which several treatments are compared, without other factors interfering with the outcome. It is desirable to have comparative doubleblinded RCTs but hitherto all comparative studies are retrospective studies or open, prospective studies. Nevertheless, these studies have supplied us with relevant information about efficacy and speed of conventional systemic therapy versus biologics, and about efficacy of conventional and anti-TNF $\alpha$ therapy compared with other drugs in the same group. Secukinumab and ustekinumab were not included in any of these studies; therefore, the relative effectiveness of these anti-IL-17 and anti-IL-12/23 biologics remain to be seen.

The results of a prospective study comparing anti-TNF biologics with acitretin, methotrexate, and narrowband (NB)-UVB phototherapy draws attention to the importance of adequate follow-up [80]. The short follow-up of only 16 weeks resulted in no significant change in NAPSI for any of the conventional therapies, contrary to the six patients receiving anti-TNF $\alpha$. On the other hand, this study also showed that patience is more important when using conventional therapies than using biologics. These differences in speed between biologics and conventional treatments (with the exception of cyclosporine) were also noted by Sanchez-Regana et al. [79], whose retrospective study compared phototherapy and several systemic and biologic therapies: acitretin, methotrexate, cyclosporine, PUVA, NBUVB, retinoid (RE)-PUVA, RE-NB-UVB, infliximab, efalizumab, etanercept, or adalimumab [79]. Significant reductions were identified in the mean NAPSI scores at 12 , 24 , and 48 weeks for all antipsoriatic agents, with the exception of NB-UVB. In the group treated with conventional systemic drugs, nail psoriasis improvement was significantly higher in patients treated with cyclosporine $(p<0.01)$ than in those treated with acitretin, methotrexate, PUVA, and RE-NUVB at 12 and 24 weeks. In the biological treatment group, differences between distinct biological treatments had disappeared at 48 weeks, although the percentage of reduction in the NAPSI score was significantly greater with infliximab and adalimumab at 12 and 24 weeks. Cyclosporine was as effective as infliximab and adalimumab. Similar efficacy of cyclosporine and adalimumab was also concluded from a prospective comparison of adalimumab with cyclosporine in PsA patients also suffering from nail psoriasis [84]. Patients were treated with cyclosporine, adalimumab (40 mg every other week), or a combination of cyclosporine and adalimumab. After 12 months of treatment, NAPSI-50 was achieved in $44 \%$ of patients receiving cyclosporine, $56 \%$ of patients receiving adalimumab, and $100 \%$ of patients receiving a combination of the two drugs.

Other comparative studies included only biologics. Two open, randomized, prospective studies investigated infliximab, etanercept, and adalimumab in a limited number of patients $[125,126]$. After 48 weeks of treatment, Ozmen et al. reported a reduction in NAPSI of 40,57 , and $54 \%$ in patients receiving infliximab, etanercept, and adalimumab, respectively (not significant) [125]. Saraceno et al. studied the efficacy and differences in efficacy of the same three anti-TNF $\alpha$ treatments in 60 patients with moderate nail psoriasis [126]. At 24 weeks (the end of follow-up in this study), no significant differences between the three biologics could be measured, but mean NAPSI had reduced to $91 \%$ in infliximab-treated patients, $70 \%$ in etanercepttreated patients, and $66 \%$ in adalimumab-treated patients. However, at week 14, efficacy was higher in the infliximab group compared with the adalimumab and etanercept groups. NAPSI-75 could be achieved in 65,45 , and $50 \%$ of patients treated for 24 weeks with infliximab, etanercept, and adalimumab, respectively. The lack of major differences in efficacy on nail psoriasis can also be concluded from several daily practice and retrospective studies [122-124]. Sola-Ortigosa et al. measured a NAPSI improvement of $57 \%$ in 15 patients after 24 weeks of adalimumab treatment [122], while the study of Bardazzi et al. in 16 patients receiving adalimumab showed a better NAPSI response at this time point $(-89 \%)$, and $94 \%$ NAPSI improvement at 36 weeks follow-up [123]. The third retrospective study comparing the efficacy of anti$\mathrm{TNF} \alpha$ biologics on nail psoriasis also failed to show differences between infliximab, etanercept, and adalimumab in 39 patients [124]. After 48 weeks of treatment, a reduction in NAPSI of 95,92 , and $87 \%$, respectively, was observed. One retrospective study focusing on determining which biologic is the most effective in the treatment of nail psoriasis also included ustekinumab [123]. At 36 weeks, no significant differences were seen in the reduction of NAPSI, and the percentage of patients achieving NAPSI$75(89 \%)$ was also similar in all groups.

The efficacy of biologics has not only been a matter of research but differences in the occurrence of adverse events have also been investigated. A prospective, randomized, open-label study compared the incidence of onychomycosis in 315 patients with nail psoriasis treated with infliximab, etanercept, and adalimumab, and a statistically positive association between fungal infections and the use of infliximab was shown [103]. At the end of week 24, $33 \%$ of patients receiving infliximab had a positive fungal culture. In patients receiving etanercept and adalimumab, and controls, this percentage was $15.5,13.3$, and $13.9 \%$, 
respectively. On the other hand, NAPSI improved more $(86 \%)$ in infliximab-treated patients than in patients receiving etanercept (68\%) and adalimumab (71\%).

The preliminary conclusions that can be drawn from these studies are that conventional therapies are less powerful and are slower-acting treatments for nail psoriasis than biologics, possibly with the exception of cyclosporine. No single biologic appears to be superior above another biologic. Evidence suggests that infliximab may act faster, but after 1 year of treatment these potential differences have vanished. On the other hand, the use of infliximab may result in higher percentages of onychomycosis.

\section{Non-Pharmacological Treatment Options}

\subsection{Laser Therapy}

Laser treatment is an emerging physical therapy option claiming an indication in increasing numbers of skin and nail disorders. In nail psoriasis, PDL $(595 \mathrm{~nm})$ treatments are also offered. A positive clinical effect of the laser is supposed to be caused by its effect on angiogenesis and vascularity within the psoriatic nail unit. Several case reports and clinical studies have been reported [57, 151155], and the results of these reports are rather contradictory. While some authors claim effects mainly on nail bed psoriasis [155], others see more positive results on nail matrix psoriasis [57, 152-154], or even negative effects on nail bed psoriasis [57]. The reported NAPSI improvement by laser therapy ranges from a disappointing $2 \%$ at 6 months [57] to a sensational $86 \%$ at 1 month [155]. In addition, a rapid decrease in NAPSI, followed by a significant increase after the third month of treatment despite ongoing treatment, has been reported [154]. Several protocols to treat nail psoriasis with PDL have been advocated but comparisons did not show significant differences between 6-ms pulse duration, $9 \mathrm{~J} / \mathrm{cm}^{2}, 7 \mathrm{~mm}$ spot size, and $0.45-\mathrm{ms}, 6 \mathrm{~J} / \mathrm{cm}^{2}, 7 \mathrm{~mm}$ spot size [152, 154]. Combining PDL with photodynamic therapy [153] or tazarotene [57] has been studied. Additional photodynamic therapy with methylaminolevulinic acid or tazarotene did not significantly change the NAPSI results; however, a significantly higher percentage of patients had improvement in PGA and SGA scores after 6 months of treatment with topical tazarotene plus PDL than after tazarotene treatment alone.

The major side effect of PDL treatment is pain, which may last for $24 \mathrm{~h}$. Other adverse events are petechiae and hyperpigmentation in $30 \%$ of the nail folds, i.e. in virtually all patients. Pain, but also petechiae, is worse if a longer pulse duration is chosen. Summarizing these often conflicting results with PDL, one must conclude that a randomized study comparing PDL treatment with sham laser treatment is urgently needed to justify this painful treatment in patients with nail psoriasis.

\subsection{Phototherapy: Topical Phototherapy, Systemic Phototherapy, Photodynamic Therapy}

Phototherapy refers to a therapeutic technique based on the beneficial effect of light waves on several pathological conditions. In dermatology, the light of several wavelengths are used (UVA, UVB, visible light), sometimes in combination with oral or topical photosensitizers (psoralens in PUVA, or aminolevulinic acid in photodynamic therapy). All these phototherapeutic options have been investigated in nail psoriasis. Photodynamic therapy has been shown to be ineffective in nail psoriasis, both as monotherapy and as a pretreatment in PDL [153]. PUVA phototherapy with either oral or topical psoralens appears to be effective and beneficial in both nail bed and nail matrix disease [79, 156, 157]. In a small, retrospective study, adding an oral retinoid to PUVA has been shown to increase NAPSI improvement from 69 to $85 \%$ [79]. NBUVB therapy, as well as with the Excimer laser, appears to be much less effective, or not effective at all, in treating nail psoriasis $[79,80,158]$. The positive results of UVA and NB-UVB are surprising considering that the mean penetration of UVA light through the fingernails is only $1.65 \%$, and UVB is completely blocked [159]. A major problem with PUVA is the risk of development of nonmelanoma skin cancer after prolonged treatment.

\subsection{Radiotherapy}

Radiotherapy is rarely used in the daily clinical care of patients with nail psoriasis. Nevertheless, cases and clinical trials with several radiotherapeutical modalities have been reported [160-164], resulting in the inclusion of these treatments in the conclusions of the Cochrane review on the treatment of nail psoriasis [15]. Efficacy of superficial radiotherapy [160, 162, 164], Grenz ray therapy [161], and electron beam therapy [163] was confirmed in case reports and RCTs. Improvement of nail psoriasis was generally limited and only temporary, and local hyperpigmentation can be seen with most of these treatments. Safety concerns are another reason why radiotherapy cannot be recommended as a first-choice therapy for the long-term treatment of nail psoriasis.

\section{Conclusions}

Growing attention for the impaired QoL and high association with potential joint-damaging PsA has resulted in increased attention for nail psoriasis. Almost all recent 
clinical trials on biologics have included nail psoriasis as a secondary endpoint, and all anti-TNF $\alpha$, anti-IL-17, and anti-IL-12/23 antibodies studied have shown slow but excellent response for psoriatic nail disease; however, topical, intralesional, and conventional systemic treatments have also proven their value for patients with nail psoriasis. The important heterogeneity in design, outcome measure, baseline disease, and follow-up make it virtually impossible to conduct a meta-analysis, or to even compare the results of the studies.

The optimal treatment for a patient depends on many individual factors, including the impact on QoL, disease severity, nail bed or nail matrix disease, number of involved nails, patience and motivation, concomitant arthritis, and other comorbidities. The hierarchy of treatments comprises topical, intralesional, and systemic treatments. Topical treatments are often slowly acting but helpful in mild to moderate cases in patients who can adhere to the treatment for a long period. Corticosteroids may be more effective in nail matrix psoriasis than in nail bed psoriasis, while calcipotriol shows an inverse profile. Intralesional steroids may be the second step and, considering the painful procedure, are mainly indicated in patients with only a few involved fingernails. Conventional systemic therapy, including newer small molecules, are indicated in therapy-resistant nail psoriasis, with or without PP and PsA. Its efficacy on nail psoriasis appears to be less than, and slower than, that of biologics, but cyclosporine may be a more powerful and faster treatment option than the other conventional systemic therapies. Biologics may be on the highest step of the hierarchical ladder. All biologics available for PP or PsA appear to have excellent results on the nail, apparently without major differences between individual biologics or the epitope they target. In addition, non-pharmacological treatment options of radiotherapy, PUVA, and RE-PUVA have proven their value, but the disadvantage of these treatment options is the risk of adverse events, making long-term treatment unattractive. Better studies are needed to justify the use of laser treatment.

A major wish for the future is the development and implementation of a reliable core set of outcome measures for future trials in nail psoriasis in order to obtain data that will make it possible to compare data.

\section{Compliance with Ethical Standards}

Marcel C. Pasch has received research support from Pfizer (support paid to institution) and has sat on advisory boards for Amgen and Celgene (honorarium paid to institution). No funding or assistance was received for the preparation of this manuscript.

Open Access This article is distributed under the terms of the Creative Commons Attribution-NonCommercial 4.0 International
License (http://creativecommons.org/licenses/by-nc/4.0/), which permits any noncommercial use, distribution, and reproduction in any medium, provided you give appropriate credit to the original author(s) and the source, provide a link to the Creative Commons license, and indicate if changes were made.

\section{References}

1. Jiaravuthisan MM, Sasseville D, Vender RB, Murphy F, Muhn CY. Psoriasis of the nail: anatomy, pathology, clinical presentation, and a review of the literature on therapy. J Am Acad Dermatol. 2007;57(1):1-27.

2. Klaassen KM, van de Kerkhof PC, Pasch MC. Nail psoriasis: a questionnaire-based survey. Br J Dermatol. 2013;169(2):314-9.

3. Williamson L, Dalbeth N, Dockerty JL, Gee BC, Weatherall R, Wordsworth BP. Extended report: nail disease in psoriatic arthritis-clinically important, potentially treatable and often overlooked. Rheumatology (Oxford). 2004;43(6):790-4.

4. Salomon J, Szepietowski JC, Proniewicz A. Psoriatic nails: a prospective clinical study. J Cutan Med Surg. 2003;7(4):317-21.

5. Maejima H, Taniguchi T, Watarai A, Katsuoka K. Evaluation of nail disease in psoriatic arthritis by using a modified nail psoriasis severity score index. Int J Dermatol. 2010;49(8):901-6.

6. Langenbruch A, Radtke MA, Krensel M, Jacobi A, Reich K, Augustin M. Nail involvement as a predictor of concomitant psoriatic arthritis in patients with psoriasis. $\mathrm{Br} \mathrm{J}$ Dermatol. 2014;171(5):1123-8.

7. Heydendael VM, de Borgie CA, Spuls PI, Bossuyt PM, Bos JD, de Rie MA. The burden of psoriasis is not determined by disease severity only. J Investig Dermatol Symp Proc. 2004;9(2):131-5.

8. Fortune DG, Main CJ, O'Sullivan TM, Griffiths CE. Quality of life in patients with psoriasis: the contribution of clinical variables and psoriasis-specific stress. $\mathrm{Br} \mathrm{J}$ Dermatol. 1997;137(5):755-60.

9. Rapp SR, Feldman SR, Exum ML, Fleischer AB Jr, Reboussin DM. Psoriasis causes as much disability as other major medical diseases. J Am Acad Dermatol. 1999;41(3 Pt 1):401-7.

10. Krueger G, Koo J, Lebwohl M, Menter A, Stern RS, Rolstad T. The impact of psoriasis on quality of life: results of a 1998 National Psoriasis Foundation patient-membership survey. Arch Dermatol. 2001;137(3):280-4.

11. Touw CR, Hakkaart-Van Roijen L, Verboom P, Paul C, Rutten FF, Finlay AY. Quality of life and clinical outcome in psoriasis patients using intermittent cyclosporin. $\mathrm{Br} \mathrm{J}$ Dermatol. 2001;144(5):967-72.

12. van der Velden HM, Klaassen KM, van de Kerkhof PC, Pasch MC. The impact of fingernail psoriasis on patients' health-related and disease-specific quality of life. Dermatology. 2014;229(2):76-82.

13. Luger TA, Barker J, Lambert J, Yang S, Robertson D, Foehl J, et al. Sustained improvement in joint pain and nail symptoms with etanercept therapy in patients with moderate-to-severe psoriasis. J Eur Acad Dermatol Venereol. 2009;23(8):896-904.

14. Klaassen KM, van de Kerkhof PC, Pasch MC. Nail psoriasis, the unknown burden of disease. J Eur Acad Dermatol Venereol. 2014;28(12):1690-5.

15. de Vries AC, Bogaards NA, Hooft L, Velema M, Pasch M, Lebwohl M, et al. Interventions for nail psoriasis. Cochrane Database Syst Rev. 2013;1:CD007633.

16. Nestle FO, Kaplan DH, Barker J. Psoriasis. N Engl J Med. 2009;361(5):496-509.

17. Mak RK, Hundhausen C, Nestle FO. Progress in understanding the immunopathogenesis of psoriasis. Actas Dermosifiliogr. 2009;100(Suppl 2):2-13. 
18. Gupta R, Debbaneh MG, Liao W. Genetic epidemiology of psoriasis. Curr Dermatol Rep. 2014;3(1):61-78.

19. Gudjonsson JE, Karason A, Antonsdottir AA, Runarsdottir EH, Gulcher JR, Stefansson K, et al. HLA-Cw6-positive and HLACw6-negative patients with psoriasis vulgaris have distinct clinical features. J Invest Dermatol. 2002;118(2):362-5.

20. Hollox EJ, Huffmeier U, Zeeuwen PL, Palla R, Lascorz J, Rodijk-Olthuis D, et al. Psoriasis is associated with increased beta-defensin genomic copy number. Nat Genet. 2008;40(1):23-5.

21. de Cid R, Riveira-Munoz E, Zeeuwen PL, Robarge J, Liao W, Dannhauser EN, et al. Deletion of the late cornified envelope LCE3B and LCE3C genes as a susceptibility factor for psoriasis. Nat Genet. 2009;41(2):211-5.

22. Jansen PA, Rodijk-Olthuis D, Hollox EJ, Kamsteeg M, Tjabringa GS, de Jongh GJ, et al. Beta-defensin-2 protein is a serum biomarker for disease activity in psoriasis and reaches biologically relevant concentrations in lesional skin. PLoS One. 2009;4(3):e4725.

23. Rich P, Scher RK. Nail psoriasis severity index: a useful tool for evaluation of nail psoriasis. J Am Acad Dermatol. 2003;49(2):206-12.

24. Klaassen KM, van de Kerkhof PC, Bastiaens MT, Plusje LG, Baran RL, Pasch MC. Scoring nail psoriasis. J Am Acad Dermatol. 2014;70(6):1061-6.

25. Augustin M, Blome C, Costanzo A, Dauden E, Ferrandiz C, Girolomoni G, et al. Nail assessment in psoriasis and psoriatic arthritis (NAPPA): development and validation of a tool for assessment of nail psoriasis outcomes. $\mathrm{Br} \mathrm{J}$ Dermatol. 2014;170(3):591-8.

26. Manhart R, Rich P. Nail psoriasis. Clin Exp Rheumatol. 2015;33(5 Suppl 93):7-13.

27. de Berker D. Management of psoriatic nail disease. Semin Cutan Med Surg. 2009;28(1):39-43.

28. Klaassen KM, Dulak MG, van de Kerkhof PC, Pasch MC. The prevalence of onychomycosis in psoriatic patients: a systematic review. J Eur Acad Dermatol Venereol. 2014;28(5):533-41.

29. Kole L, Cantrell W, Elewski B. A randomized, double-blinded trial evaluating the efficacy and tolerability of vectical ointment (calcitriol $3 \mathrm{mcg} / \mathrm{g}$ ointment) when compared to betamethasone diproprionate ointment $(64 \mathrm{mg} / \mathrm{g})$ in patients with nail psoriasis. J Drugs Dermatol. 2014;13(8):912-5.

30. Tosti A, Piraccini BM, Cameli N, Kokely F, Plozzer C, Cannata GE, et al. Calcipotriol ointment in nail psoriasis: a controlled double-blind comparison with betamethasone dipropionate and salicylic acid. Br J Dermatol. 1998;139(4):655-9.

31. Rigopoulos D, Gregoriou S, Katsambas A. Treatment of psoriatic nails with tazarotene cream $0.1 \%$ vs. clobetasol propionate $0.05 \%$ cream: a double-blind study. Acta Derm Venereol. 2007;87(2):167-8.

32. Baran R, Tosti A. Topical treatment of nail psoriasis with a new corticoid-containing nail lacquer formulation. J Dermatol Treat. 1999;10:201-4.

33. Sanchez Regana M, Martin Ezquerra G, Umbert Millet P, Llambi Mateos F. Treatment of nail psoriasis with $8 \%$ clobetasol nail lacquer: positive experience in 10 patients. J Eur Acad Dermatol Venereol. 2005;19(5):573-7.

34. Nakamura RC, Abreu L, Duque-Estrada B, Tamler C, Leverone AP. Comparison of nail lacquer clobetasol efficacy at $0.05 \%$, $1 \%$ and $8 \%$ in nail psoriasis treatment: prospective, controlled and randomized pilot study. An Bras Dermatol. 2012;87(2):203-11.

35. Rigopoulos D, Ioannides D, Prastitis N, Katsambas A. Nail psoriasis: a combined treatment using calcipotriol cream and clobetasol propionate cream. Acta Derm Venereol. 2002;82(2):140.
36. Tzung TY, Chen CY, Yang CY, Lo PY, Chen YH. Calcipotriol used as monotherapy or combination therapy with betamethasone dipropionate in the treatment of nail psoriasis. Acta Derm Venereol. 2008;88(3):279-80.

37. Rigopoulos D, Gregoriou S, Daniel CR III, Belyayeva H, Larios $\mathrm{G}$, Verra P, et al. Treatment of nail psoriasis with a two-compound formulation of calcipotriol plus betamethasone dipropionate ointment. Dermatology. 2009;218(4):338-41.

38. Korver JE, van de Kerkhof PC, Pasch MC. Alefacept treatment of psoriatic nail disease: how severe should nail psoriasis be? J Am Acad Dermatol. 2006;54(4):742-3.

39. Sanchez Regana M, Martin Ezquerra G, Umbert Millet P. Nail psoriasis: a combined treatment with $8 \%$ clobetasol nail lacquer and tacalcitol ointment. J Eur Acad Dermatol Venereol. 2008;22(8):963-9.

40. Gerstein W. Psoriasis and lichen planus of nalis. Treatment with triamcinolone. Arch Dermatol. 1962;86:419-21.

41. Abell E, Samman PD. Intradermal triamcinolone treatment of nail dystrophies. Br J Dermatol. 1973;89(2):191-7.

42. Bleeker JJ. Intralesional triamcinolone acetonide using the PortO-Jet and needle injections in localized dermatoses. Br J Dermatol. 1974;91(1):97-101.

43. Peachey RD, Pye RJ, Harman RR. The treatment of psoriatic nail dystrophy with intradermal steroid injections. Br J Dermatol. 1976;95(1):75-8.

44. de Berker DA, Lawrence CM. A simplified protocol of steroid injection for psoriatic nail dystrophy. $\mathrm{Br} \mathrm{J}$ Dermatol. 1998;138(1):90-5.

45. Saleem K, Azim W. Treatment of nail psoriasis with a modified regimen of steroid injections. J Coll Physicians Surg Pak. 2008;18(2):78-81.

46. Wolf R, Tur E, Brenner S. Corticosteroid-induced 'disappearing digit'. J Am Acad Dermatol. 1990;23(4 Pt 1):755-6.

47. Deffer TA, Goette DK. Distal phalangeal atrophy secondary to topical steroid therapy. Arch Dermatol. 1987;123(5):571-2.

48. Bjorkman A, Jorgsholm P. Rupture of the extensor pollicis longus tendon: a study of aetiological factors. Scand J Plast Reconstr Surg Hand Surg. 2004;38(1):32-5.

49. Jakubik J. Finger tendon rupture following local application of triamcinolone-acetonide (Kenalog A-40). Acta Chir Plast. 1981;23(3):180-8.

50. Usmani N, Wilson C. A case of nail psoriasis treated with topical calcitriol. Clin Exp Dermatol. 2006;31(5):712-3.

51. Marquez Balbas G, Sanchez Regana M, Martin Ezquerra G, Umbert Millet P. Tacalcitol ointment for the treatment of nail psoriasis. J Dermatolog Treat. 2009;20(5):308-10.

52. Zakeri M, Valikhani M, Mortazavi H, Barzegari M. Topical calcipotriol therapy in nail psoriasis: a study of 24 cases. Dermatol Online J. 2005;11(3):5.

53. Feliciani C, Zampetti A, Forleo P, Cerritelli L, Amerio P, Proietto G, et al. Nail psoriasis: combined therapy with systemic cyclosporin and topical calcipotriol. J Cutan Med Surg. 2004;8(2):122-5.

54. Scher RK, Stiller M, Zhu YI. Tazarotene $0.1 \%$ gel in the treatment of fingernail psoriasis: a double-blind, randomized, vehicle-controlled study. Cutis. 2001;68(5):355-8.

55. Bianchi L, Soda R, Diluvio L, Chimenti S. Tazarotene $0.1 \%$ gel for psoriasis of the fingernails and toenails: an open, prospective study. Br J Dermatol. 2003;149(1):207-9.

56. Fischer-Levancini C, Sanchez-Regana M, Llambi F, Collgros H, Exposito-Serrano V, Umbert-Millet P. Nail psoriasis: treatment with tazarotene $0.1 \%$ hydrophilic ointment. Actas Dermosifiliogr. 2012;103(8):725-8.

57. Huang YC, Chou CL, Chiang YY. Efficacy of pulsed dye laser plus topical tazarotene versus topical tazarotene alone in 
psoriatic nail disease: a single-blind, intrapatient left-to-right controlled study. Lasers Surg Med. 2013;45(2):102-7.

58. Diluvio L, Campione E, Paterno EJ, Mordenti C, El Hachem M, Chimenti S. Childhood nail psoriasis: a useful treatment with tazarotene $0.05 \%$. Pediatr Dermatol. 2007;24(3):332-3.

59. Heydendael VM, Spuls PI, Opmeer BC, de Borgie CA, Reitsma JB, Goldschmidt WF, et al. Methotrexate versus cyclosporine in moderate-to-severe chronic plaque psoriasis. N Engl J Med. 2003;349(7):658-65.

60. Tosti A, Guerra L, Bardazzi F, Lanzarini M. Topical ciclosporin in nail psoriasis. Dermatologica. 1990;180(2):110.

61. Cannavo SP, Guarneri F, Vaccaro M, Borgia F, Guarneri B. Treatment of psoriatic nails with topical cyclosporin: a prospective, randomized placebo-controlled study. Dermatology. 2003;206(2):153-6.

62. De Simone C, Maiorino A, Tassone F, D'Agostino M, Caldarola G. Tacrolimus $0.1 \%$ ointment in nail psoriasis: a randomized controlled open-label study. J Eur Acad Dermatol Venereol. 2013;27(8):1003-6.

63. Hermann RC, Taylor RS, Ellis CN, Williams NA, Weiner ND, Flynn GL, et al. Topical ciclosporin for psoriasis: in vitro skin penetration and clinical study. Skin Pharmacol. 1988;1(4):246-9.

64. Prins AM, Vos K, Franssen EJ. Instability of topical ciclosporin emulsion for nail psoriasis. Dermatology. 2007;215(4):362-3.

65. Sehgal VN, Verma P, Khurana A. Anthralin/dithranol in dermatology. Int J Dermatol. 2014;53(10):e449-60.

66. Yamamoto T, Katayama I, Nishioka K. Topical anthralin therapy for refractory nail psoriasis. J Dermatol. 1998;25(4):231-3.

67. Fredriksson T. Topically applied fluorouracil in the treatment of psoriatic nails. Arch Dermatol. 1974;110(5):735-6.

68. Fritz K. Successful local treatment of nail psoriasis with 5-fluorouracil [in German]. Z Hautkr. 1989;64(12):1083-8.

69. de Jong EM, Menke HE, van Praag MC, van De Kerkhof PC. Dystrophic psoriatic fingernails treated with $1 \% 5$-fluorouracil in a nail penetration-enhancing vehicle: a double-blind study. Dermatology. 1999;199(4):313-8.

70. Feuerman EJ, Nir MA. Allopurinol in psoriasis: a double-blind study. Br J Dermatol. 1973;89(1):83-6.

71. Saricaoglu H, Oz A, Turan H. Nail psoriasis successfully treated with intralesional methotrexate: case report. Dermatology. 2011;222(1):5-7.

72. Lassus A. Colloidal silicic acid for the treatment of psoriatic skin lesions, arthropathy and onychopathy. A pilot study. J Int Med Res. 1997;25(4):206-9.

73. Lin YK, Chang CJ, Chang YC, Wong WR, Chang SC, Pang JH. Clinical assessment of patients with recalcitrant psoriasis in a randomized, observer-blind, vehicle-controlled trial using indigo naturalis. Arch Dermatol. 2008;144(11):1457-64.

74. Lin YK, See LC, Chang YC, Huang YH, Chen JL, Tsou TC, et al. Treatment of psoriatic nails with indigo naturalis oil extract: a non-controlled pilot study. Dermatology. 2011;223(3):239-43.

75. Lin YK, Chang YC, Hui RC, See LC, Chang CJ, Yang CH, et al. A Chinese herb, indigo naturalis, extracted in oil (Lindioil) used topically to treat psoriatic nails: a randomized clinical trial. JAMA Dermatol. 2015;151(6):672-4.

76. Lin YK, See LC, Huang YH, Chang YC, Tsou TC, Lin TY, et al. Efficacy and safety of Indigo naturalis extract in oil (Lindioil) in treating nail psoriasis: a randomized, observer-blind, vehiclecontrolled trial. Phytomedicine. 2014;21(7):1015-20.

77. Gumusel M, Ozdemir M, Mevlitoglu I, Bodur S. Evaluation of the efficacy of methotrexate and cyclosporine therapies on psoriatic nails: a one-blind, randomized study. J Eur Acad Dermatol Venereol. 2011;25(9):1080-4.
78. Reich K, Langley RG, Papp KA, Ortonne JP, Unnebrink K, Kaul $\mathrm{M}$, et al. A 52-week trial comparing briakinumab with methotrexate in patients with psoriasis. $\mathrm{N}$ Engl $\mathrm{J}$ Med. 2011;365(17):1586-96.

79. Sanchez-Regana M, Sola-Ortigosa J, Alsina-Gibert M, VidalFernandez M, Umbert-Millet P. Nail psoriasis: a retrospective study on the effectiveness of systemic treatments (classical and biological therapy). J Eur Acad Dermatol Venereol. 2011;25(5):579-86.

80. Demirsoy EO, Kiran R, Salman S, Caglayan C, Akturk AS, Bayramgurler D, et al. Effectiveness of systemic treatment agents on psoriatic nails: a comparative study. J Drugs Dermatol. 2013;12(9):1039-43.

81. Arnold WP, Gerritsen MJ, van de Kerkhof PC. Response of nail psoriasis to cyclosporin. Br J Dermatol. 1993;129(6):750-1.

82. Mahrle G, Schulze HJ, Farber L, Weidinger G, Steigleder GK. Low-dose short-term cyclosporine versus etretinate in psoriasis: improvement of skin, nail, and joint involvement. J Am Acad Dermatol. 1995;32(1):78-88.

83. Syuto T, Abe M, Ishibuchi H, Ishikawa O. Successful treatment of psoriatic nails with low-dose cyclosporine administration. Eur J Dermatol. 2007;17(3):248-9.

84. Karanikolas GN, Koukli EM, Katsalira A, Arida A, Petrou D, Komninou E, et al. Adalimumab or cyclosporine as monotherapy and in combination in severe psoriatic arthritis: results from a prospective 12-month nonrandomized unblinded clinical trial. J Rheumatol. 2011;38(11):2466-74.

85. Dogra S, Yadav S. Acitretin in psoriasis: an evolving scenario. Int J Dermatol. 2014;53(5):525-38.

86. Ricceri F, Pescitelli L, Tripo L, Bassi A, Prignano F. Treatment of severe nail psoriasis with acitretin: an impressive therapeutic result. Dermatol Ther. 2013;26(1):77-8.

87. Tosti A, Ricotti C, Romanelli P, Cameli N, Piraccini BM. Evaluation of the efficacy of acitretin therapy for nail psoriasis. Arch Dermatol. 2009;145(3):269-71.

88. Deeks ED. Apremilast: a review in psoriasis and psoriatic arthritis. Drugs. 2015;75(12):1393-403.

89. Papp K, Reich K, Leonardi CL, Kircik L, Chimenti S, Langley RG, et al. Apremilast, an oral phosphodiesterase 4 (PDE4) inhibitor, in patients with moderate to severe plaque psoriasis: results of a phase III, randomized, controlled trial (efficacy and safety trial evaluating the effects of apremilast in psoriasis [ESTEEM] 1). J Am Acad Dermatol. 2015;73(1):37-49.

90. Paul C, Cather J, Gooderham M, Poulin Y, Mrowietz U, Ferrandiz $\mathrm{C}$, et al. Efficacy and safety of apremilast, an oral phosphodiesterase 4 inhibitor, in patients with moderate-to-severe plaque psoriasis over 52 weeks: a phase III, randomized controlled trial (ESTEEM 2). Br J Dermatol. 2015;173(6):1387-99.

91. Rich P, Gooderham M, Bachelez H, Goncalves J, Day RM, Chen R, et al. Apremilast, an oral phosphodiesterase 4 inhibitor, in patients with difficult-to-treat nail and scalp psoriasis: results of 2 phase III randomized, controlled trials (ESTEEM 1 and ESTEEM 2). J Am Acad Dermatol. 2016;74(1):134-42.

92. Vlachou C, Berth-Jones J. Nail psoriasis improvement in a patient treated with fumaric acid esters. J Dermatol Treat. 2007;18(3):175-7.

93. Gerster JC, Hohl D. Nail lesions in psoriatic arthritis: recovery with sulfasalazine treatment. Ann Rheum Dis. 2002;61(3):277.

94. Behrens F, Finkenwirth C, Pavelka K, Stolfa J, Sipek-Dolnicar A, Thaci D, et al. Leflunomide in psoriatic arthritis: results from a large European prospective observational study. Arthritis Care Res (Hoboken). 2013;65(3):464-70.

95. Van den Bosch F, Manger B, Goupille P, McHugh N, Rodevand E, Holck P, et al. Effectiveness of adalimumab in treating patients with active psoriatic arthritis and predictors of good 
clinical responses for arthritis, skin and nail lesions. Ann Rheum Dis. 2010;69(2):394-9.

96. Rigopoulos D, Gregoriou S, Lazaridou E, Belyayeva E, Apalla Z, Makris M, et al. Treatment of nail psoriasis with adalimumab: an open label unblinded study. J Eur Acad Dermatol Venereol. 2010;24(5):530-4.

97. Pirowska MM, Gozdzialska A, Lipko-Godlewska S, Obtulowicz A, Sulowicz J, Podolec K, et al. Autoimmunogenicity during anti-TNF therapy in patients with psoriasis and psoriatic arthritis. Postepy Dermatol Alergol. 2015;32(4):250-4.

98. Callen JP. Complications and adverse reactions in the use of newer biologic agents. Semin Cutan Med Surg. 2007;26(1):6-14.

99. Gottlieb AB, Evans R, Li S, Dooley LT, Guzzo CA, Baker D, et al. Infliximab induction therapy for patients with severe plaque-type psoriasis: a randomized, double-blind, placebo-controlled trial. J Am Acad Dermatol. 2004;51(4):534-42.

100. Reich K, Nestle FO, Papp K, Ortonne JP, Evans R, Guzzo C, et al. Infliximab induction and maintenance therapy for moderate-to-severe psoriasis: a phase III, multicentre, double-blind trial. Lancet. 2005;366(9494):1367-74.

101. Menter A, Feldman SR, Weinstein GD, Papp K, Evans R, Guzzo $\mathrm{C}$, et al. A randomized comparison of continuous vs. intermittent infliximab maintenance regimens over 1 year in the treatment of moderate-to-severe plaque psoriasis. J Am Acad Dermatol. 2007;56(1):31 (e1-15)

102. Krishnan RS, Hsu S. Serum sickness due to infliximab in a patient with psoriasis. J Drugs Dermatol. 2004;3(3):305-8.

103. Al-Mutairi N, Nour T, Al-Rqobah D. Onychomycosis in patients of nail psoriasis on biologic therapy: a randomized, prospective open label study comparing etanercept, infliximab and adalimumab. Expert Opin Biol Ther. 2013;13(5):625-9.

104. Reich K, Ortonne JP, Kerkmann U, Wang Y, Saurat JH, Papp K, et al. Skin and nail responses after 1 year of infliximab therapy in patients with moderate-to-severe psoriasis: a retrospective analysis of the EXPRESS Trial. Dermatology. 2010;221(2):172-8.

105. Rigopoulos D, Gregoriou S, Stratigos A, Larios G, Korfitis C, Papaioannou D, et al. Evaluation of the efficacy and safety of infliximab on psoriatic nails: an unblinded, nonrandomized, open-label study. Br J Dermatol. 2008;159(2):453-6.

106. Rich P, Griffiths CE, Reich K, Nestle FO, Scher RK, Li S, et al. Baseline nail disease in patients with moderate to severe psoriasis and response to treatment with infliximab during 1 year. J Am Acad Dermatol. 2008;58(2):224-31.

107. Torii H, Sato N, Yoshinari T, Nakagawa H. Dramatic impact of a Psoriasis Area and Severity Index 90 response on the quality of life in patients with psoriasis: an analysis of Japanese clinical trials of infliximab. J Dermatol. 2012;39(3):253-9.

108. Torii H. Nakagawa H; Japanese Infliximab Study Investigators. Infliximab monotherapy in Japanese patients with moderate-tosevere plaque psoriasis and psoriatic arthritis. A randomized, double-blind, placebo-controlled multicenter trial. J Dermatol Sci. 2010;59(1):40-9.

109. Torii H, Nakagawa H, Japanese Infliximab Study Investigators. Long-term study of infliximab in Japanese patients with plaque psoriasis, psoriatic arthritis, pustular psoriasis and psoriatic erythroderma. J Dermatol. 2011;38(4):321-34.

110. Hussain W, Coulson I, Owen C. Severe recalcitrant nail psoriasis responding dramatically to infliximab: report of two patients. Clin Exp Dermatol. 2008;33(4):520-2.

111. Safa G, Darrieux L. Dramatic response of nail psoriasis to infliximab. Case Rep Med. 2011;2011:107928.

112. Papoutsaki M, Osorio F, Morais P, Torres T, Magina S, Chimenti $\mathrm{S}$, et al. Infliximab in psoriasis and psoriatic arthritis. BioDrugs. 2013;27(Suppl 1):13-23.
113. Fabroni C, Gori A, Troiano M, Prignano F, Lotti T. Infliximab efficacy in nail psoriasis. A retrospective study in 48 patients. J Eur Acad Dermatol Venereol. 2011;25(5):549-53.

114. Bianchi L, Bergamin A, de Felice C, Capriotti E, Chimenti S. Remission and time of resolution of nail psoriasis during infliximab therapy. J Am Acad Dermatol. 2005;52(4):736-7.

115. Irla N, Yawalkar N. Marked improvement in nail psoriasis during treatment with adalimumab. Dermatology. 2009;219(4):353-6.

116. Rudwaleit M, Van den Bosch F, Kron M, Kary S, Kupper H. Effectiveness and safety of adalimumab in patients with ankylosing spondylitis or psoriatic arthritis and history of anti-tumor necrosis factor therapy. Arthritis Res Ther. 2010;12(3):R117.

117. Thaci D, Unnebrink K, Sundaram M, Sood S, Yamaguchi Y. Adalimumab for the treatment of moderate to severe psoriasis: subanalysis of effects on scalp and nails in the BELIEVE study. J Eur Acad Dermatol Venereol. 2015;29(2):353-60.

118. Thaci D, Ortonne JP, Chimenti S, Ghislain PD, Arenberger P, Kragballe $\mathrm{K}$, et al. A phase IIIb, multicentre, randomized, double-blind, vehicle-controlled study of the efficacy and safety of adalimumab with and without calcipotriol/betamethasone topical treatment in patients with moderate to severe psoriasis: the BELIEVE study. Br J Dermatol. 2010;163(2):402-11.

119. Leonardi C, Langley RG, Papp K, Tyring SK, Wasel N, Vender $\mathrm{R}$, et al. Adalimumab for treatment of moderate to severe chronic plaque psoriasis of the hands and feet: efficacy and safety results from REACH, a randomized, placebo-controlled, double-blind trial. Arch Dermatol. 2011;147(4):429-36.

120. Paul C, van de Kerkhof P, Puig L, Unnebrink K, Goldblum O, Thaci D. Influence of psoriatic arthritis on the efficacy of adalimumab and on the treatment response of other markers of psoriasis burden: subanalysis of the BELIEVE study. Eur $\mathrm{J}$ Dermatol. 2012;22(6):762-9.

121. Poulin Y, Crowley JJ, Langley RG, Unnebrink K, Goldblum OM, Valdecantos WC. Efficacy of adalimumab across subgroups of patients with moderate-to-severe chronic plaque psoriasis of the hands and/or feet: post hoc analysis of REACH. J Eur Acad Dermatol Venereol. 2014;28(7):882-90.

122. Sola-Ortigosa J, Sanchez-Regana M, Umbert-Millet P. Efficacy of adalimumab in the treatment of psoriasis: a retrospective study of 15 patients in daily practice. J Dermatol Treat. 2012;23(3):203-7.

123. Bardazzi F, Antonucci VA, Tengattini V, Odorici G, Balestri R, Patrizi A. A 36-week retrospective open trial comparing the efficacy of biological therapies in nail psoriasis. J Dtsch Dermatol Ges. 2013;11(11):1065-70.

124. Kyriakou A, Patsatsi A, Sotiriadis D. Anti-TNF agents and nail psoriasis: a single-center, retrospective, comparative study. J Dermatol Treat. 2013;24(3):162-8.

125. Ozmen I, Erbil AH, Koc E, Tunca M. Treatment of nail psoriasis with tumor necrosis factor-alpha blocker agents: an open-label, unblinded, comparative study. J Dermatol. 2013;40(9):755-6.

126. Saraceno R, Pietroleonardo L, Mazzotta A, Zangrilli A, Bianchi L, Chimenti S. TNF-alpha antagonists and nail psoriasis: an open, 24-week, prospective cohort study in adult patients with psoriasis. Expert Opin Biol Ther. 2013;13(4):469-73.

127. Leonardi CL, Powers JL, Matheson RT, Goffe BS, Zitnik R, Wang A, et al. Etanercept as monotherapy in patients with psoriasis. N Engl J Med. 2003;349(21):2014-22.

128. Rallis E, Stavropoulou E, Rigopoulos D, Verros C. Rapid response of nail psoriasis to etanercept. J Rheumatol. 2008;35(3):544-5.

129. Coelho JD, Diamantino F, Lestre S, Ferreira AM. Treatment of severe nail psoriasis with etanercept. Indian J Dermatol Venereol Leprol. 2011;77(1):72-4. 
130. Gomez Vazquez M, Navarra Amayuelas R. Marked improvement in nail psoriasis during treatment with etanercept. Dermatol Ther. 2011;24(5):498-500.

131. Barrera MV, Habicheyn S, Mendiola MV, Herrera Ceballos E. Etanercept in the treatment and retreatment of psoriasis in daily clinical practice. Eur J Dermatol. 2008;18(6):683-7.

132. Ortonne JP, Paul C, Berardesca E, Marino V, Gallo G, Brault Y, et al. A 24-week randomized clinical trial investigating the efficacy and safety of two doses of etanercept in nail psoriasis. Br J Dermatol. 2013;168(5):1080-7.

133. Kavanaugh A, McInnes I, Mease P, Krueger GG, Gladman D, Gomez-Reino J, et al. Golimumab, a new human tumor necrosis factor alpha antibody, administered every four weeks as a subcutaneous injection in psoriatic arthritis: twenty-four-week efficacy and safety results of a randomized, placebo-controlled study. Arthritis Rheum. 2009;60(4):976-86.

134. Kavanaugh A, van der Heijde D, McInnes IB, Mease P, Krueger GG, Gladman DD, et al. Golimumab in psoriatic arthritis: oneyear clinical efficacy, radiographic, and safety results from a phase III, randomized, placebo-controlled trial. Arthritis Rheum. 2012;64(8):2504-17.

135. Mease PJ, Fleischmann R, Deodhar AA, Wollenhaupt J, Khraishi M, Kielar D, et al. Effect of certolizumab pegol on signs and symptoms in patients with psoriatic arthritis: 24 -week results of a phase 3 double-blind randomised placebo-controlled study (RAPID-PsA). Ann Rheum Dis. 2014;73(1):48-55.

136. Chiricozzi A, Krueger JG. IL-17 targeted therapies for psoriasis. Expert Opin Investig Drugs. 2013;22(8):993-1005.

137. Papp KA, Langley RG, Sigurgeirsson B, Abe M, Baker DR, Konno $\mathrm{P}$, et al. Efficacy and safety of secukinumab in the treatment of moderate-to-severe plaque psoriasis: a randomized, double-blind, placebo-controlled phase II dose-ranging study. Br J Dermatol. 2013;168(2):412-21.

138. Rich P, Sigurgeirsson B, Thaci D, Ortonne JP, Paul C, Schopf $\mathrm{RE}$, et al. Secukinumab induction and maintenance therapy in moderate-to-severe plaque psoriasis: a randomized, doubleblind, placebo-controlled, phase II regimen-finding study. Br J Dermatol. 2013;168(2):402-11.

139. Paul C, Reich K, Gottlieb AB, Mrowietz U, Philipp S, Nakayama $\mathrm{J}$, et al. Secukinumab improves hand, foot and nail lesions in moderate-to-severe plaque psoriasis: subanalysis of a randomized, double-blind, placebo-controlled, regimen-finding phase 2 trial. J Eur Acad Dermatol Venereol. 2014;28(12):1670-5.

140. Spuls PI, Hooft L. Brodalumab and ixekizumab, anti-interleukin-17-receptor antibodies for psoriasis: a critical appraisal. Br J Dermatol. 2012;167(4):710-3 (discussion 4-5).

141. Wu JJ. Anti-interleukin-17 monoclonal antibody ixekizumab in psoriasis. N Engl J Med. 2012;367(3):274-5 (author reply 5).

142. Leonardi C, Matheson R, Zachariae C, Cameron G, Li L, EdsonHeredia E, et al. Anti-interleukin-17 monoclonal antibody ixekizumab in chronic plaque psoriasis. $\mathrm{N}$ Engl $\mathrm{J}$ Med. 2012;366(13):1190-9.

143. Langley RG, Rich P, Menter A, Krueger G, Goldblum O, Dutronc Y, et al. Improvement of scalp and nail lesions with ixekizumab in a phase 2 trial in patients with chronic plaque psoriasis. J Eur Acad Dermatol Venereol. 2015;29(9):1763-70.

144. Tzellos T, Kyrgidis A, Zouboulis CC. Re-evaluation of the risk for major adverse cardiovascular events in patients treated with anti-IL-12/23 biological agents for chronic plaque psoriasis: a meta-analysis of randomized controlled trials. J Eur Acad Dermatol Venereol. 2013;27(5):622-7.

145. Rallis E, Kintzoglou S, Verros C. Ustekinumab for rapid treatment of nail psoriasis. Arch Dermatol. 2010;146(11):1315-6.

146. Vitiello M, Tosti A, Abuchar A, Zaiac M, Kerdel FA. Ustekinumab for the treatment of nail psoriasis in heavily treated psoriatic patients. Int J Dermatol. 2013;52(3):358-62.
147. Rigopoulos D, Gregoriou S, Makris M, Ioannides D. Efficacy of ustekinumab in nail psoriasis and improvement in nail-associated quality of life in a population treated with ustekinumab for cutaneous psoriasis: an open prospective unblinded study. Dermatology. 2011;223(4):325-9.

148. Patsatsi A, Kyriakou A, Sotiriadis D. Ustekinumab in nail psoriasis: an open-label, uncontrolled, nonrandomized study. J Dermatol Treat. 2013;24(2):96-100.

149. Igarashi A, Kato T, Kato M, Song M, Nakagawa H. Efficacy and safety of ustekinumab in Japanese patients with moderate-tosevere plaque-type psoriasis: long-term results from a phase $2 / 3$ clinical trial. J Dermatol. 2012;39(3):242-52.

150. Rich P, Bourcier M, Sofen H, Fakharzadeh S, Wasfi Y, Wang Y, et al. Ustekinumab improves nail disease in patients with moderate-to-severe psoriasis: results from PHOENIX 1. Br J Dermatol. 2014;170(2):398-407.

151. Al-Mutairi A, Elkashlan M. Nail psoriasis treated with pulse dye laser. Indian J Dermatol. 2013;58(3):243.

152. Goldust M, Raghifar R. Clinical trial study in the treatment of nail psoriasis with pulsed dye laser. J Cosmet Laser Ther. 2013. doi:10.3109/14764172.2013.854627.

153. Fernandez-Guarino M, Harto A, Sanchez-Ronco M, GarciaMorales I, Jaen P. Pulsed dye laser vs. photodynamic therapy in the treatment of refractory nail psoriasis: a comparative pilot study. J Eur Acad Dermatol Venereol. 2009;23(8):891-5.

154. Treewittayapoom C, Singvahanont P, Chanprapaph K, Haneke E. The effect of different pulse durations in the treatment of nail psoriasis with 595-nm pulsed dye laser: a randomized, doubleblind, intrapatient left-to-right study. J Am Acad Dermatol. 2012;66(5):807-12.

155. Oram Y, Karincaoglu Y, Koyuncu E, Kaharaman F. Pulsed dye laser in the treatment of nail psoriasis. Dermatol Surg. 2010;36(3):377-81.

156. Marx JL, Scher RK. Response of psoriatic nails to oral photochemotherapy. Arch Dermatol. 1980;116(9):1023-4.

157. Handfield-Jones SE, Boyle J, Harman RR. Local PUVA treatment for nail psoriasis. Br J Dermatol. 1987;116(2):280-1.

158. Aubin F, Vigan M, Puzenat E, Blanc D, Drobacheff C, Deprez $\mathrm{P}$, et al. Evaluation of a novel 308-nm monochromatic excimer light delivery system in dermatology: a pilot study in different chronic localized dermatoses. $\mathrm{Br}$ J Dermatol. 2005;152(1):99-103.

159. Stern DK, Creasey AA, Quijije J, Lebwohl MG. UV-A and UV$B$ penetration of normal human cadaveric fingernail plate. Arch Dermatol. 2011;147(4):439-41.

160. Finnerty EF. Successful treatment of psoriasis of the nails. Cutis. 1979;23(1):43-4.

161. Lindelof B. Radiotherapy is rarely used in daily clinical care of patients suffering from nail psoriasis. Acta Derm Venereol. 1989;69(1):80-2.

162. Yu RC, King CM. A double-blind study of superficial radiotherapy in psoriatic nail dystrophy. Acta Derm Venereol. 1992;72(2):134-6.

163. Kwang TY, Nee TS, Seng KT. A therapeutic study of nail psoriasis using electron beams. Acta Derm Venereol. 1995;75(1):90.

164. Rados J, Dobric I, Pasic A, Lipozencic J, Ledic-Drvar D, Stajminger G. Normalization in the appearance of severly damaged psoriatic nails using soft X-rays. Acta Dermatovenerol Croat. 2007;15(1):27-32.

165. Nantel-Battista M, Richer V, Marcil I, Benohanian A. Treatment of nail psoriasis with intralesional triamcinolone acetonide using a needle-free jet injector: a prospective trial. J Cutan Med Surg. 2014;18(1):38-42. 